\title{
MicroRNA-155 inhibits the osteogenic differentiation of mesenchymal stem cells induced by BMP9 via downregulation of BMP signaling pathway
}

\author{
HONGXIA LIU ${ }^{1}$, LIANG ZHONG ${ }^{1}$, TAIXIAN YUAN ${ }^{1}$, SICHENG CHEN ${ }^{2}$, YIQING ZHOU ${ }^{1}$, LIQIN AN ${ }^{1}$, \\ YANGLIU GUO ${ }^{1}$, MENGTIAN FAN $^{1}$, YA LI $^{1}$, YANTING SUN ${ }^{1}$, WANG LI ${ }^{1}$, QIONG SHI ${ }^{1}$ and YAGUANG WENG ${ }^{1}$ \\ ${ }^{1}$ Key Laboratory of Diagnostic Medicine Designated by the Chinese Ministry of Education, Chongqing Medical University, \\ Chongqing 400016; ${ }^{2}$ Xinxiang Medical University, Xinxiang, Henan 453002, P.R. China
}

Received December 19, 2016; Accepted February 12, 2018

DOI: $10.3892 / \mathrm{ijmm} .2018 .3526$

\begin{abstract}
Previous studies have indicated that bone morphogenetic protein 9 (BMP9) can promote the osteogenic differentiation of mesenchymal stem cells (MSCs) and increase bone formation in bone diseases. However, the mechanisms involved remained poorly understood. It is necessary to investigate the specific regulatory mechanisms of osteogenic differentiation that were induced by BMP9. During the process of osteogenic differentiation induced by BMP9, the expression of microRNA-155 (miR-155) exhibited a tendency of increasing at first and then decreasing, which made us consider that miR-155 may have a modulatory role in this process, but the roles of this process have not been elucidated. This study aimed to uncover miR-155 capable of concomitant regulation of this process. mmu-miR-155 mimic (miR-155) was transfected into MSCs and osteogenesis was induction by using recombinant adenovirus expressing BMP9. Overexpressed miR-155 in MSCs led to a decrease in alkaline phosphatase (ALP) staining and Alizarin red S staining during osteogenic differentiation, and reduced the expression of osteogenesis-related genes, such as runt-related transcription factor 2 (Runx2), osterix (OSX), osteocalcin (OCN) and osteopontin (OPN). On protein levels, overexpressed miR-155 markedly decreased the expression of phosphorylated Smad1/5/8 (p-Smad1/5/8), Runx2, OCN and OPN. Luciferase reporter assay revealed Runx2 and bone morphogenetic protein receptor 9 (BMPR2) are two direct target genes of miR-155. Downregulation of the expression of Runx2 and BMPR2, respectively could offset the inhibitory effect of miR-155 in the osteogenesis of MSCs. In vivo, subcutaneous ectopic osteogenesis of MSCs in nude
\end{abstract}

Correspondence to: Dr Qiong Shi or Professor Yaguang Weng, Key Laboratory of Diagnostic Medicine Designated by the Chinese Ministry of Education, Chongqing Medical University, Chongqing 400016, P.R. China

E-mail: anniesq8718@aliyun.com

E-mail: yaguangweng@126.com

Key words: miR-155, bone morphogenetic protein 9, osteogenic differentiation, mesenchymal stem cells mice showed miR-155 inhibited osteogenic differentiation. In conclusion, our results demonstrated that miR-155 can inhibit the osteogenic differentiation induced by BMP9 in MSCs.

\section{Introduction}

During the process of bone regeneration, three main phases, inflammation, new bone formation and bone remodeling take place in an orchestrated fashion to repair the injury. Bone remodeling is regulated by both the number and activity of osteoblasts and osteoclasts. Osteoblast lineage commitment, proliferation and differentiation are controlled by a welldefined genetic program. Bone morphogenetic proteins (BMPs) are unique extracellular multifunctional signaling cytokines belonging to the large transforming growth factor- $\beta$ (TGF- $\beta$ ) superfamily (1-6). BMPs can induce heterogeneous oligomerization of type I and II receptor serine/threonine kinases and Smad1/5/8 phosphorylation. Subsequently, the phosphorylated Smad1/5/8 are associated with Smad4 and translocate into the nucleus where they cooperate with tissue-specific transcription factors to drive osteogenic target gene expression (7). Or through the non-canonical Smad-independent signaling [p38 mitogen-activated protein kinase (MAPK)] pathway to active downstream transcription factors to potentiate the osteoblastogenesis role (8). Bone morphogenetic protein 9 (BMP9), a member of the BMP family, in previous studies was identified it as one of the most osteogenic BMPs $(1,4,6,9,10)$, but the mechanisms involved remain largely unclear.

MicroRNAs (miRNAs or miRs) are a class of endogenous noncoding RNAs, 22 nucleotides in length that are involved in the regulation of gene expression, coordinating a broad spectrum of biological processes $(11,12)$. miRNAs influence the expression of the target gene by binding to its 3'-untranslated region (3'-UTR) and inactivate it by promoting its degradation or translational repression $(13,14)$. Several studies indicated that miRNAs act as key regulators in cell differentiation (15-20), cell growth $(21,22)$, cell death (23), and lipid metabolism (24). Previous studies have shown that miR-206, $-204,-133,-135,-125 \mathrm{~b},-141,-200 \mathrm{a}$ and -214 can inhibit osteogenic differentiation $(12,16,25-30)$. On the contrary, miR-15b and miR-29b have been demonstrated to promote 
osteogenesis $(31,32)$. Apparently, miRNAs are important regulators during the osteogenic differentiation. As a member of the oncomir class of microRNAs, miR-155 is implicated in lymphomagenesis (33-36) and a wide array of nonlymphoid tumors including breast, colon, and lung (33,37-41).

In recent years, several studies have focused on miRNAs being modulated by BMP signaling as a means to investigate the role of miRNAs in osteoblasts $(17,26,28,29,42)$. Another study indicated that suppressing the expression and function of miR-155 contributes to mitigate the inhibition of TNF- $\alpha$ on BMP-2-induced osteogenic differentiation (43), indicating that there was a link between miR-155 and BMP signaling. From the early studies we found that during the process of osteogenic differentiation induced by BMP9, the expression of miR-155 has changed. Overexpression of miR-155 in C2C12 myoblasts and mouse embryonic fibroblasts (MEF), induced osteogenesis by BMP9, the expression of osteogenesis-related genes like runt-related transcription factor 2 (Runx2) and alkaline phosphatase (ALP) declined, so we reasonably hypothesized that miR-155 can inhibit this process, but the relevant mechanisms are not clear. The aim of this work is to explore the effect of miR-155 on the osteogenic differentiation induced by BMP9, and investigate the correlation mechanisms.

\section{Materials and methods}

Cell lines and culture. The HEK-293 (human embryonic kidney) cell line, $\mathrm{C} 2 \mathrm{C} 12$ (myoblasts) cell line, and MEF cell line were obtained from American Type Culture Collection (ATCC, Manassas, VA, USA). The HEK-293 cells, C2C12 cells and MEF cells were all immortalized and maintained in DMEM, supplemented with $10 \%$ fetal bovine serum (FBS) (Gibco, Grand Island, NY, USA), $100 \mathrm{U} / \mathrm{ml}$ penicillin and $100 \mu \mathrm{g} / \mathrm{ml}$ streptomycin. The cells were maintained at $37^{\circ} \mathrm{C}$ in a humidified $5 \% \mathrm{CO}_{2}$ atmosphere.

Transfection of the miR-155 mimic, miR-155 inhibitor, and shBMPR2. miR-155 mimic, miR-155 inhibitor, and shBMPR2 were purchased from GenePharma (Shanghai, China). The sequences for each oligonucleotide are listed in Table I. Entranster $^{\mathrm{TM}}$ R4000 (Engreen Biosystems, Beijing, China) was utilized to transfect miR-155 mimic, miR-155 inhibitor and miR-155 negative control (NC) according to the manufacturer's instructions. To transfect shBMPR2 and it negative control shNC, Lipofectamine 2000 transfection reagent (Invitrogen, Shanghai, China) was used according to the manufacturer's instructions. In this study, $\mathrm{C} 2 \mathrm{C} 12$ cells and MEF cells were transfected with with $100 \mathrm{nM}$ of miR-155 mimic, or miR-155 inhibitor or NC in a 24-well plate or $500 \mathrm{nM}$ of the above in a 6-well plate. In a 6-well plate, shNC or shBMPR2 were transfected into cells at $800 \mathrm{ng}$ each well.

Transfection of the BMP9 and si-Runx2. C2C12 cells and MEF cells were cultured in 24-well plates, each well was added with $500 \mu 1$ DMEM supplemented with $10 \%$ FBS, and before using BMP9 and si-Runx2, we tested the titer of these recombinant adenoviruses. Each well contained $3 \mu \mathrm{l}$ of polybrene to contribute to the adenovirus entry into cells, different volumes of adenovirus was added, until the infection efficiency reached $30 \%$, the volume of the adenovirus was the
Table I. Oligoribonucleotides used in the study.

\begin{tabular}{lc}
\hline Name & Sequence $\left(5^{\prime} \rightarrow 3^{\prime}\right)$ \\
\hline mmu-miR-155 mimic & F: UUAAUGCUAAUUGUGAUAGGGGU \\
& R:CCCUAUCACAAUUAGCAUUAAUU \\
mmu-miR-155 inhibitor & F: ACCCCUAUCACAAUUAGCAUUAA \\
Negative control (NC) & F: UUCUCCGAACGUGUCACGUTT \\
& R:ACGUGACACGUUCGGAGAATT \\
shBMPR2 & F: CACCGCCAAGATGAATACAATCAA \\
& TTTCAAGAGAATTGATTGTATTCAT \\
& CTTGGCTTTTTG \\
& R:GATCCAAAAAAGCCAAGATGAATA \\
& CAATCAATTCTCTTGAAATTGATTG \\
& TATTCATCTTGGC \\
& F: CACCGTTCTCCGAACGTGTCACGTT \\
TCAAGAGAACGTGACACGTTC \\
shNC & GGAGAATTTTTG \\
& R:GATCCAAAAAATTCTCCGAACGTG \\
& TCACGTTCTCTTGAAACGTGACA \\
& CGTTCGGAGAAC
\end{tabular}

F, forward; R, reverse. BMPR2, bone morphogenetic protein receptor 2.

titer for the corresponding cells, respectively. In a 6-well plate, $12 \mu \mathrm{l}$ of polybrene and four-fold titer volume of adenovirus were added after that the cells attached.

$R N A$ extraction, reverse transcription and real-time quantitative PCR (RT-qPCR) analysis. Total RNA was extracted from $\mathrm{C} 2 \mathrm{C} 12$ cells and MEF cells at the indicated time points using TRIzol reagent (Ambion, Aukland, New Zealand) according to the manufacturer's instructions for the analysis of mRNA and miRNA expression. The concentration and purity of RNA were quantified using spectrophotometer (NanoDrop 1000 Spectrophotometer; Thermo Fisher Scientific Inc., Waltham, MA, USA). The extracted total RNA (2,000 ng) was reverse transcribed using reverse transcription primers according to the manufacturer's instructions (Reverse Transcriptase M-MLV, Takara code: D2639A; Takara Bio Inc., Otsu, Japan). The primers used for reverse transcription PCR (RT-PCR) and RT-qPCR are listed in Table II. RT-qPCR were performed on a Bio-Rad iQ5 instrument (Bio-Rad, Hercules, CA, USA) used cDNA as templates to amplify target genes, mixing with SYBR ${ }^{\circledR}$-Green PCR Master Mix (Takara Bio, Inc.), Foldchanges in expression were calculated using the $2^{-\Delta \Delta C t}$ method, and each experiment was performed in triplicate. Data were analyzed using Optical system software, version 2.0. The expression levels of $\beta$-actin and the small U6 RNA were used as internal controls for mRNA and miRNA, respectively.

$A L P$ staining and ALP activity. ALP staining was performed with BCIP/NBT Alkaline Phosphatase Color Development kit (C3206; Beyotime, Shanghai, China) according to the manufacturer's instructions. Briefly, we seeded the $\mathrm{C} 2 \mathrm{C} 12$ cells and MEF cells in 24-well plates followed by treatment with NC, miR-155 mimic or miR-155 inhibitor and BMP9 for 7 days. The supernatant was discarded, cells were washed three times 
Table II. Primers used in the study.

Genes

Sequence $\left(5^{\prime} \rightarrow 3^{\prime}\right)$

mmu-miR-155

U6

mmu-miR-155

U6

Mouse Runx2

Mouse OSX

Mouse ALP

Mouse OCN

Mouse $\beta$-actin

Mouse BMPR2

\section{RT: GTCGTATCCAGTGCAGGGTCCGAGGTATTCGCACTGGATACGACACCCCT}

RT: AAAATATGGAACGCTTCACGAATTTG

F: GGCGTTAATGCTAATTGTGAT

F: CTCGCTTCGGCAGCACATATACT

F: TCTGACAAAGCCTTCATGTCC

F: GAAGTCCAATGGGGATCTGA

F: TGACCTTCTCTCCTCCATCC

F: CTGCTTGTGACGAGCTATCAG

F: ATGAAGGCGTGGCAACAT

F: GCAGTGAGGTCACTCAAGGA
R: GTGCAGGGTCCGAGGT

R:ACGCTTCACGAATTTGCGTGTC

R: AAATAGTGATACCGTAGATGCG

R: GAATCCCTTTCCCTCTCCAG

R: CTTCCTGGGAGTCTCATCCT

R: TGATACCGTAGATGCGTTTGT

R: GCCATTGGCTCTGTCCTG

R: CATCATGAGTTCAGCCATCC

RT, reverse transcription; F, forward; R, reverse; Runx2, runt-related transcription factor 2; OSX, osterix; OCN, osteocalcin; BMPR2, bone morphogenetic protein receptor 2 .

using phosphate-buffered saline (PBS). Then $3 \mathrm{ml}$ of ALP developing buffer was mixed with $10 \mu \mathrm{l}$ BCIP, and $20 \mu \mathrm{l}$ NBT to form BCIP/NBT working liquid and $200 \mu \mathrm{BCIP} / \mathrm{NBT}$ was the working liquid in each well of 24-well plates, and the plates were placed in the dark to undergo ALP staining for $30 \mathrm{~min}$. Finally, BCIP/NBT working liquid was removed by suction and the staining observed. Cells were scanned at both a lower (ScanMaker 3600; Shanghai Microtek Technology Co., Ltd., Shanghai, China) and higher magnification under a bright field microscope (T-DH; Nikon Corp., Tokyo, Japan). ALP activity was measured using an established enzymatic assay. ALP substrate was prepared from solution A (8 ng naphtol-AS-TR phosphate/300 ml N,N-dimethylformamide; both from SigmaAldrich, St. Louis, MO, USA) and solution B (24 mg fast blue BB salt/30 $\mathrm{ml}$ of $100 \mathrm{mmol} / \mathrm{l}$ Tris $\mathrm{HCl}, \mathrm{pH}$ 9.6). The two solutions were combined, $10 \mathrm{mg}$ of $\mathrm{MgCl}_{2}$ was added, the resulting solution was used immediately. Enzymatic activity was determined in cell lysates that were solubilized with $0.1 \%$ Triton X-100. Aliquots $(5 \mu \mathrm{l})$ of each sample were incubated with $15 \mu \mathrm{l}$ of AP substrate buffer $(100 \mathrm{mmol} / \mathrm{l}$ diethanolamine, $150 \mathrm{mmol} / \mathrm{l}$ $\mathrm{NaCl}, 2 \mathrm{mmol} / 1 \mathrm{MgCl}_{2}$ p-nitrophenylphosphate at $2.5 \mathrm{mg} / \mathrm{ml}$ ) and $5 \mu \mathrm{l}$ of ALP substrate under the conditions of protection from light for 30 to $40 \mathrm{~min}$ at room temperature. ALP activity was tested using a luminometer (Promega, Madison, WI, USA).

Alizarin red S staining. C2C12 cells and MEF cells were seeded in 24-well plates treated with NC, miR-155 mimic or miR-155 inhibitor and BMP9 for 14 days. The supernatant was discarded, and cells washed three times with PBS. The cells were fixed with $0.05 \%$ ice-cold glutaraldehyde (Chongqing Chuandong Chemical Group) for $10 \mathrm{~min}$ and rinsed with double-distilled $\mathrm{H}_{2} \mathrm{O}$. Then stained with $40 \mathrm{mM}(2 \%)$ Alizarin red $\mathrm{S}$ (Sigma-Aldrich) at $\mathrm{pH} 4.0$, rocked gently for $\sim 5 \mathrm{~min}$. The cells were rinsed three times with double-distilled $\mathrm{H}_{2} \mathrm{O}$ and then rinsed for 15 min with PBS and gently shaken. Finally cells were scanned at both a lower (ScanMaker 3600; Shanghai Microtek Technology Co., Ltd.) and higher magnification under a bright field microscope (T-DH; Nikon Corp.).

Western blot analysis. C2C12 cells and MEF cells were plated into a $10-\mathrm{cm}$ Petri dishes. After treating cells as before, and when cells reached $80 \%$ confluence, cell lysate was collected and protein concentrations were determined using the Bio-Rad protein assay kit. The protein $(50 \mu \mathrm{g})$ was boiled for $5 \mathrm{~min}$ in Laemmli buffer [62.5 $\mathrm{mM}$ Tris (pH 6.8), $1 \%$ sodium dodecyl sulfate (SDS), $20 \%$ glycerol, $0.01 \%$ bromophenol blue, and $100 \mathrm{mM}$ DTT] before being loaded onto a $12 \%$ SDS-polyacrylamide gel and then transferred to PVDF membranes. Specific protein bands were detected with mouse anti-BMPR2 polyclonal antibody (1:200 dilution, cat. no. BM2821; BOSTER), rabbit anti-p-Smad1/5/8 monoclonal antibody (1:1,000 dilution, cat. no. sc-12353), rabbit anti-Smad1/5/8 monoclonal antibody (1:1,000 dilution, cat. no. sc-6031-R, lot no. D1911), rabbit anti-Runx2 monoclonal antibody (1:1,000 dilution, cat. no. sc-10758, lot no. A0810) (all from Santa Cruz Biotechnology, Inc., Santa Cruz, CA, USA), rabbit anti-osteocalcin (OCN) polyclonal antibody (1:200 dilution, cat. no. bs-4917R; Beijing Biosynthesis Biotechnology Co., Ltd.), rabbit anti-osteopontin (OPN) polyclonal antibody (1:500 dilution, cat. no. wl00691; Wanleibio, Shenyang, China), or anti- $\beta$-actin mouse monoclonal antibody (1:1,000 dilution; Zhongshan Golden Bridge Biotechnology Co., Ltd., Beijing, China) overnight at $4^{\circ} \mathrm{C}$, and then washed with TBST three times, each time for $15 \mathrm{~min}$, the membranes were incubated with goat anti-rabbit or goat antimouse horseradish peroxidase conjugated secondary antibody (1:5,000 dilution; Zhongshan Golden Bridge Biotechnology Co., Ltd.), for $1 \mathrm{~h}$ at $37^{\circ} \mathrm{C}$. After that, membranes were washed with TBST three times, each time for $15 \mathrm{~min}$. The secondary antibodies were detected with western chemiluminescence reagent (Millipore Corp., Billerica, MA, USA). The results were recorded using the Bio-Rad electrophoresis documentation system (Gel Doc 1000) and Quantity One software, version 4.5.0.

Luciferase reporter assay. TargetScan (http://www.targetscan. org) and PicTar (http://pictar. mdc-berlin.de/) were utilized to identify the possible target genes of miR-155 in BMP9-induced osteogenic differentiation of $\mathrm{C} 2 \mathrm{C} 12$ cells and MEF cells. The 3'-UTR of Runx2 and BMPR2 containing miR-155-binding sequences, and the sequences contained binding sites with miR-155 were synthesised following the manufacturer's 
Table III. The sequences contained binding sites with miR-155.

\begin{tabular}{ll}
\hline Name & \multicolumn{1}{c}{ Sequence $\left(5^{\prime} \rightarrow 3^{\prime}\right)$} \\
\hline WT-Runx2 & F: CTAGGATGTAGTTTGTTTTCACAATGTATGAAGGAGATGCTCTG \\
R: AGCTCAGAGCATCTCCTTCATACATTGTGAAAACAAACTACATC & F: CTAGGATGTAGTTTGTTTAGTAATGTATGAAGGAGATGCTCTG \\
R: AGCTCAGAGCATCTCCTTCATACATTACTAAAACAAACTACATC & F: CTAGCTTATGGGGTAATTAGCATTATAAGACTTTATAAAAGTGAGCTGATGGCTCTAGC \\
WT-BMPR2 & R: AGCTGCTAGAGCCATCAGCTCACTTTTATAAAGTCTTATAATGCTAATTACCCCATAAG \\
MT-BMPR2 & F: CTAGCTTATGGGGTAATTATTAGGATAAGACTTTATAAAAGTGAGCTGATGGCTCTAGC \\
& R: AGCTGCTAGAGCCATCAGCTCACTTTTATAAAGTCTTATCCTAATAATTACCCCATAAG
\end{tabular}

WT-Runx2, Runx2-3'-UTR-wild-type; MT-Runx2, Runx2-3'-UTR-mutant type; WT-BMPR2, BMPR2-3'-UTR-wild-type; MT-BMPR2, BMPR2-3'-UTR-mutant type.

instructions (pMIR-REPORT ${ }^{\mathrm{TM}}$ system, miRNA expression reporter vector; Ambion, Carlsbad, CA, USA). The sequences contained binding sites with miR-155 are listed in Table III. The annealing fragments were then cloned into the SpeI/HindIII backbone of the pMIR-REPORT microRNA (miRNA) expression reporter (Ambion). Binding-region mutations were achieved using an oligo synthesis following the manufacturer's instructions (Ambion). Transient transfection into HEK-293 cells ( $1 \times 10^{4}$ cells/well) was carried out in 24-well plates with Lipofectamine 2000 transfection reagents (Invitrogen) following the manufacturer's instructions. The cells were co-transfected with $800 \mathrm{ng}$ of the luciferase construct plasmid and miR-155 mimic or NC, and luciferase assays were performed using the dual-luciferase reporter assay system (Ambion) according to the manufacturer's instructions. Luminescent signals were quantified using a luminometer (Promega), and each value of luciferase activity was normalized by the luciferase activity of cells that transfected empty vector only.

Ectopic in vivo bone formation assay of transfected MEF cells. All animal experiments were approved by Institutional Animal Care and Use Committee (IACUC) of Chongqing Medical University. The nude mice were purchased from Huafukang (Beijing Hfk Bioscience Co., Ltd., Beijing, China). MEF cells were transfected as described above, and the long-lasting mimic (mmu-agomiR-155) and inhibitor (mmuantagomiR-155) of miR-155 were used, and the sequences are fully consistent with mmu-miR-155 or mmu-anti-miR-155. The sequence of stable NC is the same as the NC used before. In short, after treating cells like before, cells were collected at $80 \%$ confluence and for subcutaneous injection $\left(5 \times 10^{6} \%\right.$ injection) into the flanks of the athymic nude mice (5 animals/ group, 4-6-week old, female) (44). At 4 weeks after implantation, heterotopic bones were harvested from mice after euthanasia, fixed in $4 \%$ paraformaldehyde and scanned using a $\mu \mathrm{CT} 40$ system (Scanco Medical, Brüttisellen, Switzerland). Parameters computed from these data include bone mineral density (BMD), bone volume (BV/TV), trabecular thickness (Tb.Th), trabecular number (Tb.N) and trabecular separation (Tb.Sp). For histological analysis of bones, after scanning used $\mu \mathrm{CT}$, decalcified in $10 \%$ EDTA for $\sim 4$ weeks, before being embedded in paraffin. Sections $(5 \mu \mathrm{m})$ were cut and stained with hematoxylin and eosin (H\&E) or with Masson's trichrome according to the manufacturer's instructions. Bone histomorphometry was performed using a microscope (T-DH; Nikon Corp.).

Statistical analysis. The quantitative experiments were performed in triplicate and/or repeated three times. Data are represented as the mean \pm SD. Statistical significance between the control and treatment groups were determined by one-way analysis of variance and the Student's t-test. All statistical analyses were performed using GraphPad Prism 5 (GraphPad Software, Inc., La Jolla, CA, USA). P<0.05 was considered statistically significant.

\section{Results}

During the early stage of osteogenic differentiation induced by BMP9, the expression of miR-155 was increased at first and then decreased. Several differential expression miRNAs including miR-21, miR-23b and miR-155 have been previously identified during the early stage of mesenchymal stem cells (MSCs) osteogenesis induced by BMP9 through microarray data analysis (data not shown). Allowing us to consider that there are some associations between miR-155 and BMP9 in osteogenesis. During the early stage of osteogenic differentiation induced by BMP9, we detected the expression of miR-155 by using RT-qPCR on different days. It was found that compared with the expression level of 0 day, the expression level of miR-155 was upregulated in the early stage of BMP9-induced C2C12 cells and MEF cells osteogenic differentiation, and on the 5th day reached the maximum expression, then decreased on the 7th day (Fig. 1A). In our laboratory, BMP9 contains green fluorescence protein (GFP) tag, and so the results of fluorescence microscopy proved that BMP9 was transfected into $\mathrm{C} 2 \mathrm{C} 12$ and MEF cells effectively (Fig. 1B). To further confirm the osteoinduction effect of BMP9, RT-qPCR was used to detect the mRNA expression level of osteoblast-specific genes over 7 days, including Runx 2 and ALP. The results revealed that successful osteogenic differentiation of the $\mathrm{C} 2 \mathrm{C} 12$ and MEF cells was induced (Fig. 1C).

Overexpression of miR-155 suppresses BMP9-induced ALP and calcium deposition in MSCs. Subsequently, to investigate 
A

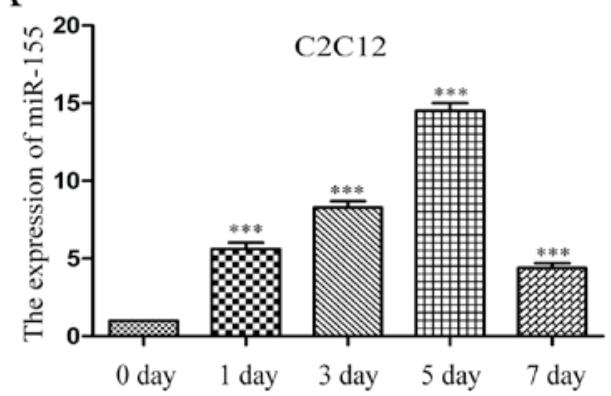

B

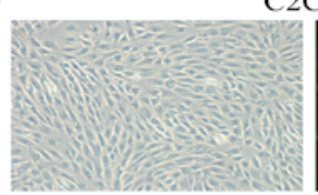

Blank
$\mathrm{C} 2 \mathrm{C} 12$

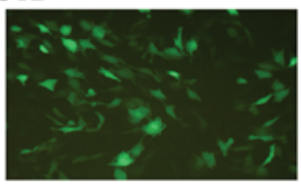

BMP9

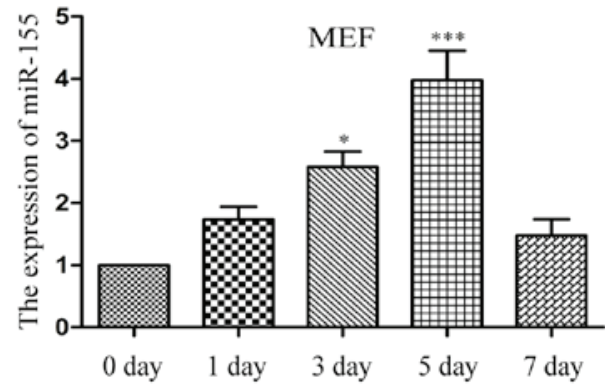

MEF

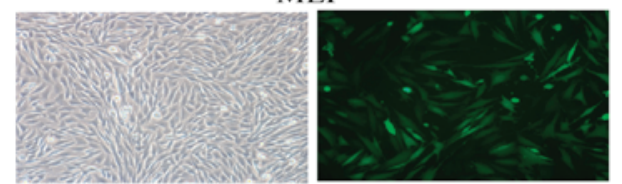

Blank
BMP9

$\mathrm{C}$
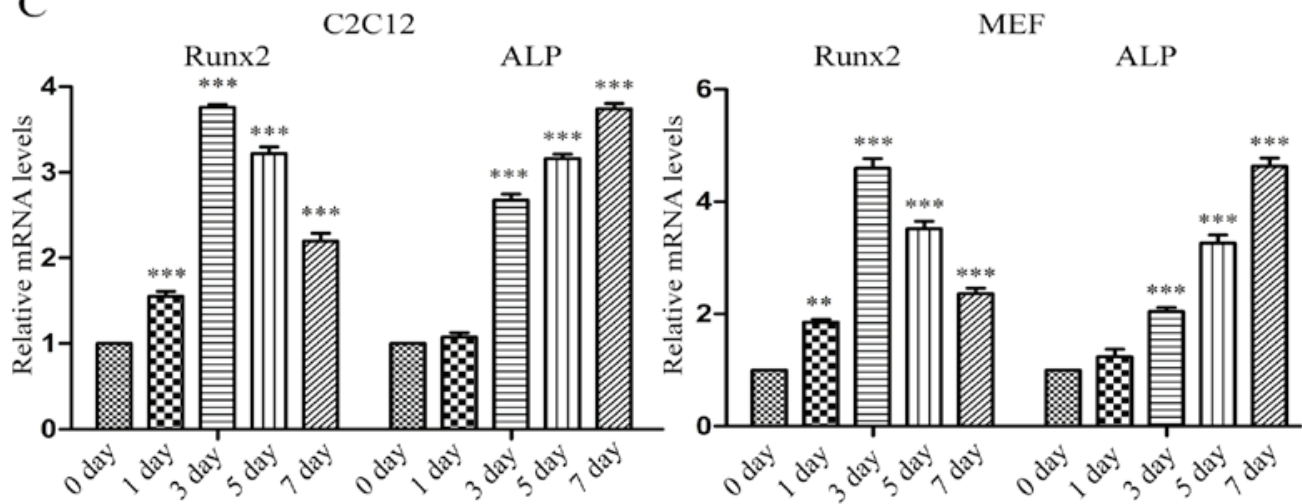

Figure 1. The expression level of miR-155 is increased at first and then decreased in the process of bone morphogenetic protein 9 (BMP9)-induced early osteogenesis. (A) The expression of miR-155 was detected by RT-qPCR. * $\mathrm{p}<0.05$ and ${ }^{* * * *} \mathrm{p}<0.001$ vs. the value at day 0 . (B) BMP9 was transfected into C2C12 cells and mouse embryonic fibroblasts (MEF) cells efficiently. Magnification, x100. White, white light used as the excitation light; green, blue light used as the excitation light. Multiple of infection (MOI): 5. (C) The expression of runt-related transcription factor 2 (Runx2) and alkaline phosphatase (ALP) in C2C12 cells and MEF cells in differentiation days induced by BMP9 were detected by RT-qPCR. ${ }^{* *}$ p $<0.01$ and ${ }^{* * *}$ p $<0.001$ vs. the value at day 0 . Day: osteogenic differentiation day.

the roles of miR-155 in the osteogenic differentiation of $\mathrm{C} 2 \mathrm{C} 12$ cells and MEF cells induced by BMP9, the expression level of miR-155 following transfection were tested by RT-qPCR, in the whole study, we set control groups contain BMP9 group and BMP9 + miR-negative control group (BMP9 + NC) (Fig. 3A). After transfecting miR-155 or anti-miR-155 into $\mathrm{C} 2 \mathrm{C} 12$ cells and MEF cells, respectively, induced osteogenesis by BMP9 for 7 days. ALP staining results showed that compared with the control groups, overexpressed miR-155 (BMP9 + miR-155) downregulated the early osteogenesis differentiation indicator-ALP activity, but inhibition of miR-155 (BMP9 + anti-miR-155) attenuated this inhibitive effect (Fig. 2A, top panel), quantitative determination of ALP activity, the results were in accordance with the ALP staining of $\mathrm{C} 2 \mathrm{C} 12$ cells and MEF cells, respectively (Fig. 2A, bottom panel).

We further analyzed the effect of miR-155 on late osteogenic differentiation by Alizarin Red S staining of matrix mineralization. The results were consistent with ALP staining, overexpressed exogenous miR-155 repressed the BMP9-induced calcium deposition of $\mathrm{C} 2 \mathrm{C} 12$ cells and MEF cells, and decreased the endogenous miR-155 adverse effect (Fig. 2B). In conclusion, the above results indicated that miR-155 impairs the BMP9-induced osteoblast lineage commitment of MSCs.

miR-155 downregulates the mRNA expression level of osteogenesis-related genes. To detect the influence of miR-155 on the osteogenesis induced by BMP9 on mRNA level, firstly, we used RT-qPCR to measure the expression of miR-155 after transfection to verified that our transfection was successful. The data showed that during osteogenic differentiation induced by BMP9, compared with control group, the intracellular miR-155 level was significantly elevated by transfection with miR-155, whereas transfected with anti-miR-155 led to distinct reduction in miR-155 content (Fig. 3A). RT-qPCR was used to evaluate the expression level of Runx 2 and osterix (OSX) on the 3rd day, the expression of ALP and OCN on the 7th day of BMP9-induced osteogenesis. Compared with the control groups, the expression of these osteogenesis markers were decreased by miR-155, conversely, anti-miR- 155 increased their expression in the process of osteogenic differentiation induced by BMP9 in $\mathrm{C} 2 \mathrm{C} 12$ cells, except the expression of OSX and ALP (Fig. 3B). The results of MEF cells were consis- 
A
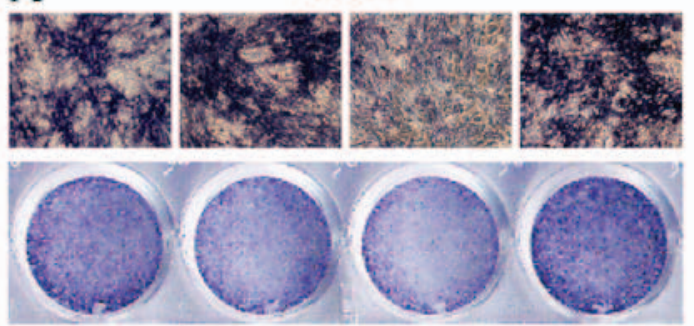

$B N P^{9}$
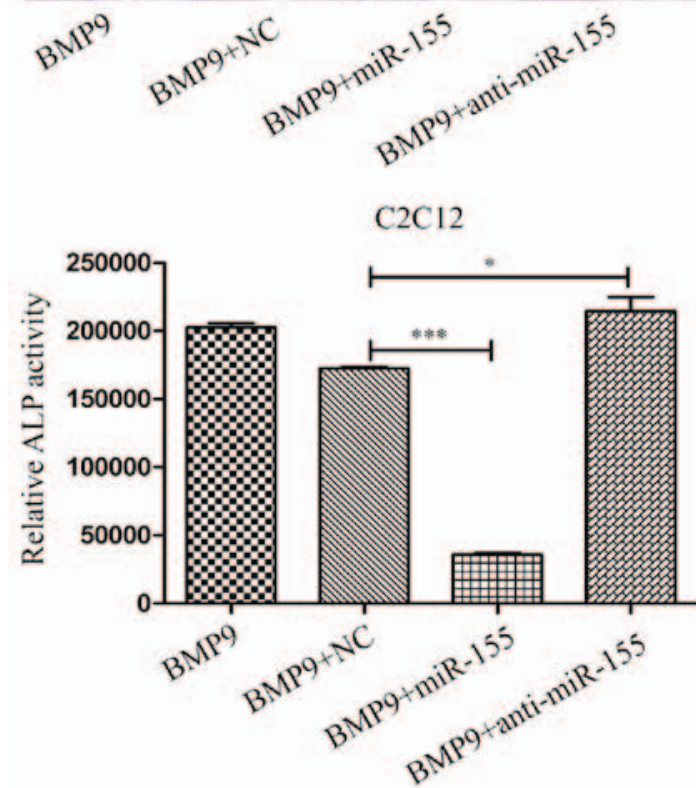

B

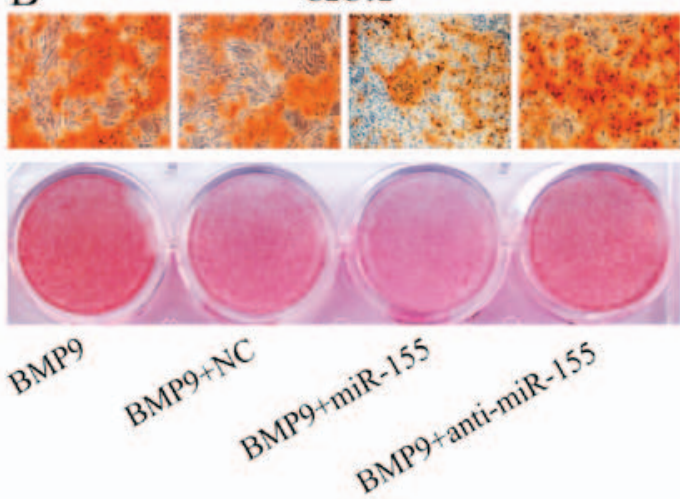

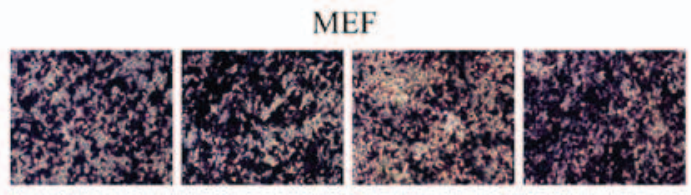
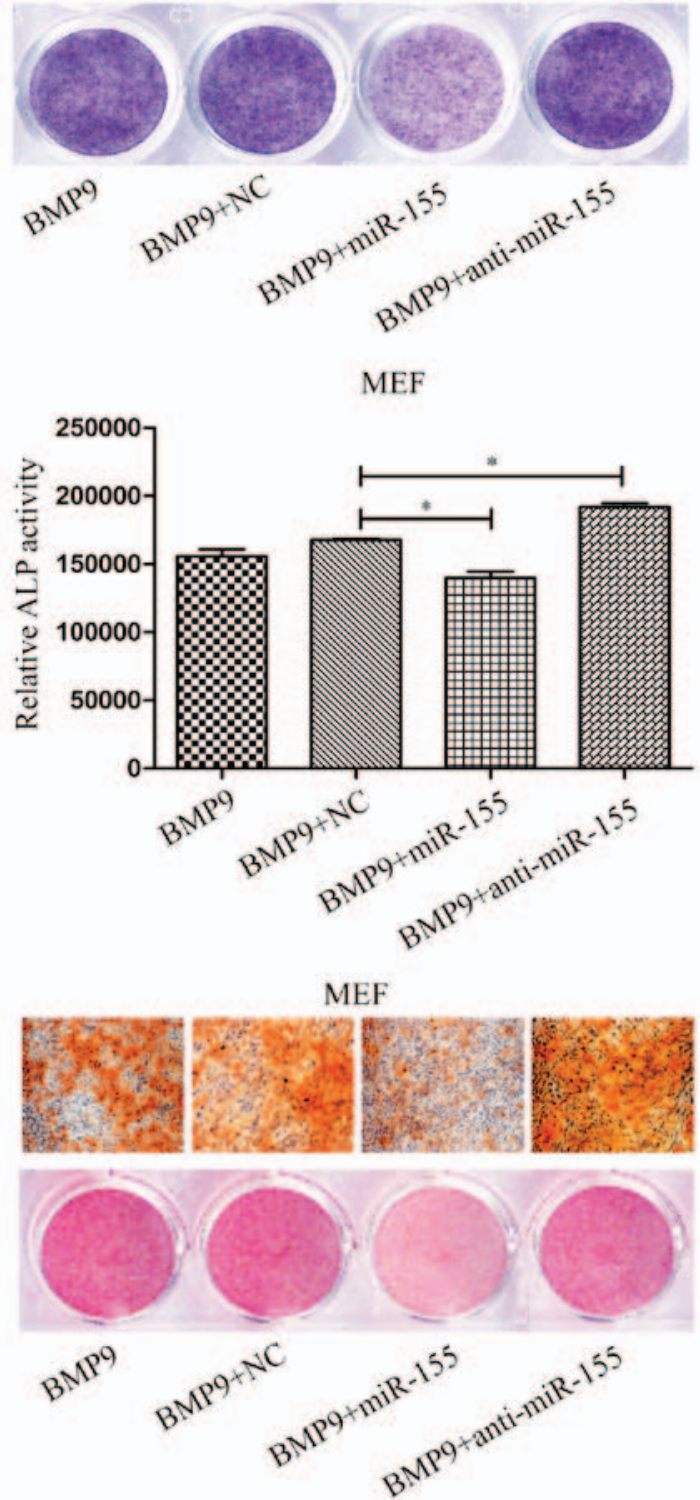

Figure 2. miR-155 inhibits the osteogenic differentiation of $\mathrm{C} 2 \mathrm{C} 12$ cells and mouse embryonic fibroblasts (MEF) cells induced by bone morphogenetic protein 9 (BMP9). (A) Alkaline phosphatase (ALP) staining and ALP activity were performed after transfecting C2C12 cells and MEF cells with NC, miR-155 or anti-miR-155, induced osteogenesis by BMP9 for 7 days. (B) The effect of miR-155 on BMP9-induced matrix mineralization though Alizarin Red S staining on the 14th day of differentiation in $\mathrm{C} 2 \mathrm{C} 12$ cells and MEF cells. Each staining was repeated three times for both types of the cells in this experiment, and one of the three independent results is shown. ${ }^{*} \mathrm{p}<0.05$ and ${ }^{* * *} \mathrm{p}<0.001$.

tent with $\mathrm{C} 2 \mathrm{C} 12$ cells (Fig. 3C). Taken together, miR-155 could suppress the expression of Runx2, OSX, ALP and OCN, which are bone differentiation related markers.

miR-155 suppresses BMP signaling during the osteogenesis induced by BMP9. Previous studies revealed that Smads, p38 and ERK1/2 are involved in BMP9-induced osteogenic differentiation (45). We hypothesized that miR-155 may through inhibiting canonical Smad-dependent signaling repress the activity of downstream transcription factors to achieve suppressed osteoblastogenesis in MSCs. The expression levels of $\mathrm{p}-\mathrm{Smad} 1 / 5 / 8$ and Runx 2 were measured by western blot analysis after treatment of cells as above. Compared with the control group, overexpressed miR-155 group (BMP9 + miR-155) diminished the expression of p-Smad1/5/8 and Runx2, however, downregulated miR-155 group (BMP9 + anti-miR-155) eliminated this inhibitory effect in C2C12 cells (Fig. 4A) and MEF cells (Fig. 5A). Based on these data, we concluded that the negative effect of miR-155 on osteogenic differentiation may be through suppressing canonical BMP (BMP/Smad) signaling.

To further examine the role of miR-155 in late stage of osteogenic differentiation, we tested the expression of $\mathrm{OCN}$ and OPN by western blot analysis after treating $\mathrm{C} 2 \mathrm{C} 12$ cells 
A

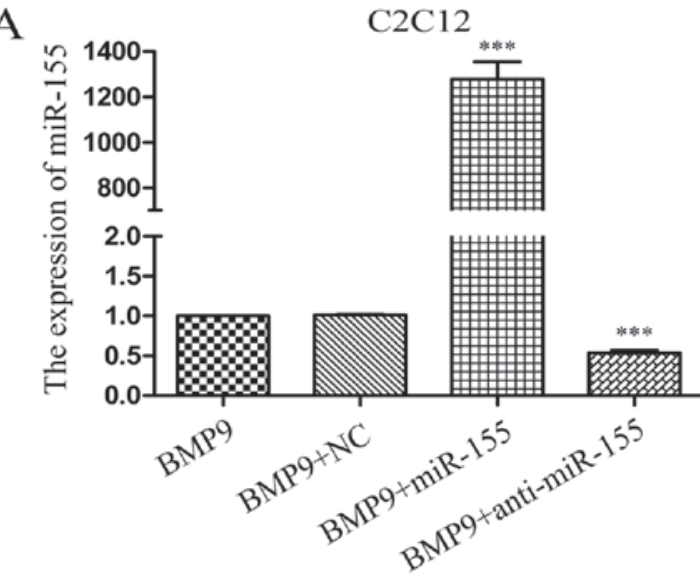

B

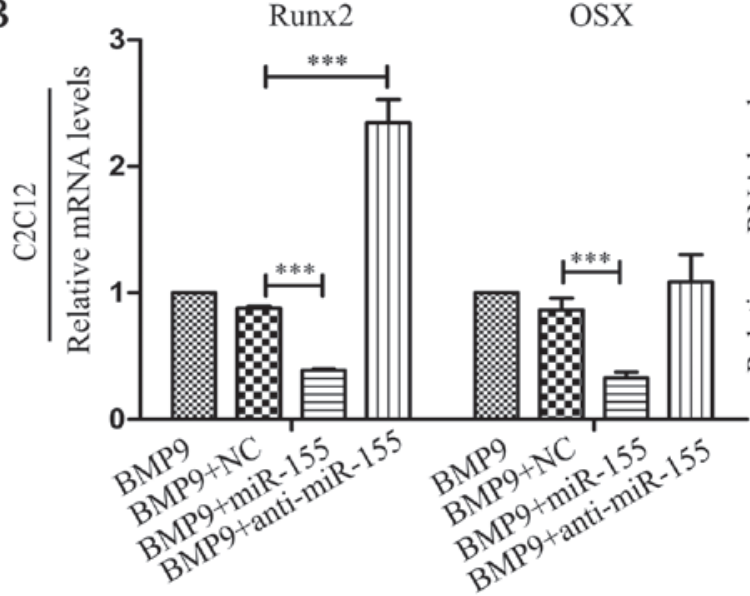

C

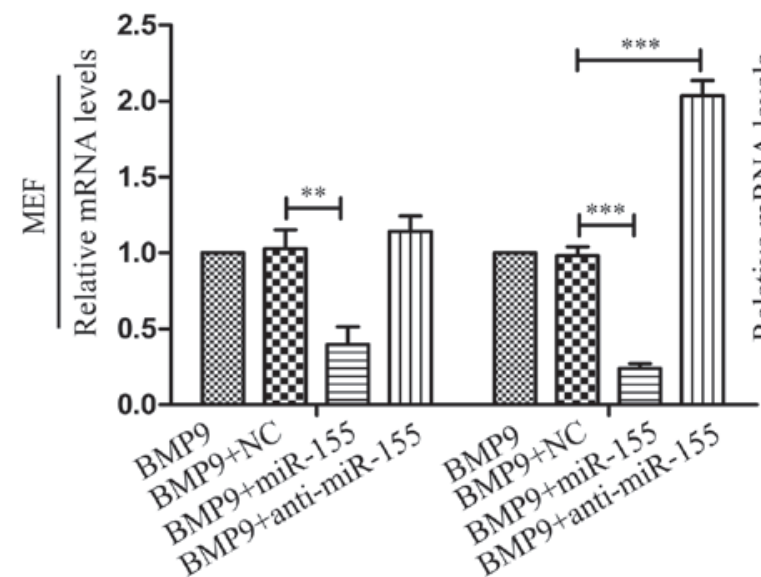

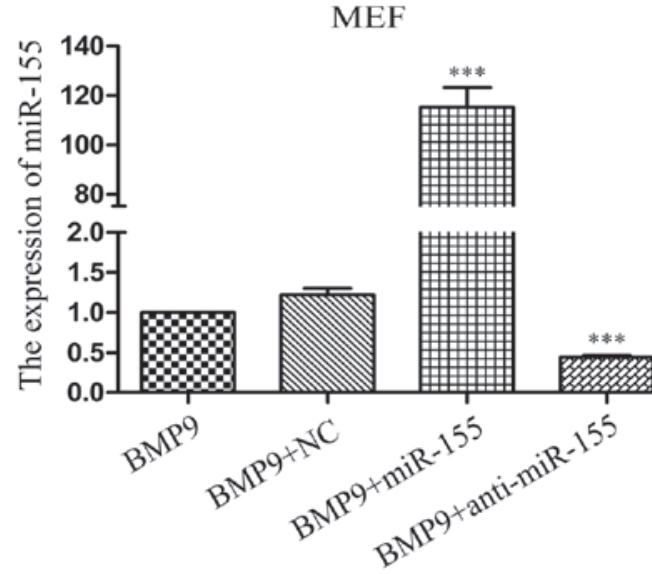

ALP

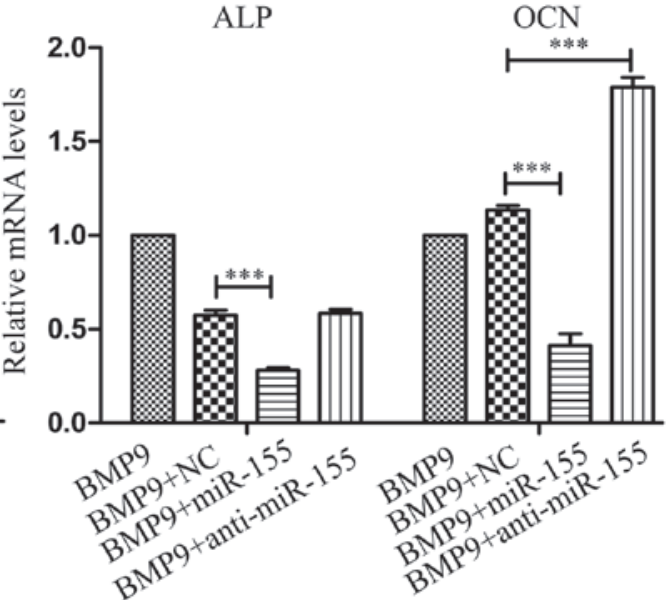

ALP

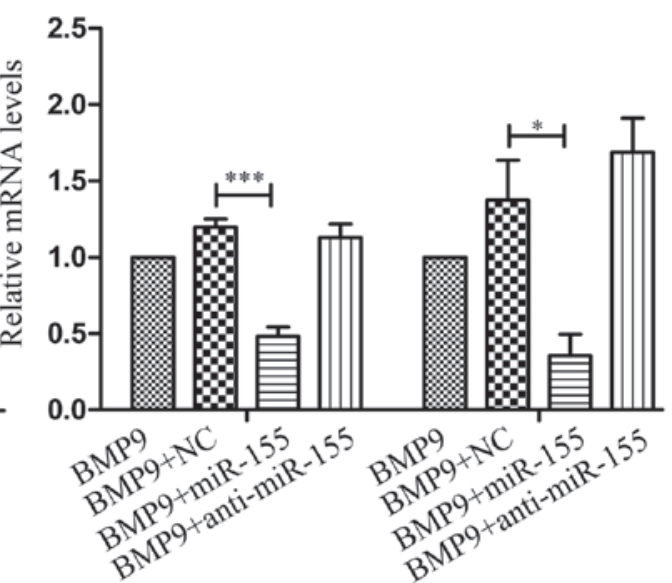

Figure 3. miR-155 downregulates the mRNA expression levels of osteogenesis genes runt-related transcription factor 2 (Runx2), osterix (OSX), alkaline phosphatase (ALP) and osteocalcin (OCN). C2C12 cells and mouse embryonic fibroblasts (MEF) were transfected with NC, miR-155 or anti-miR-155, respectively, then induced osteogenesis by bone morphogenetic protein 9 (BMP9). (A) The expression of miR-155 by RT-qPCR in the third day of differentiation. (B) Runx 2 and OSX were tested on the 3rd day of differentiation, ALP and OCN were tested on the 7th day of differentiation in C2C12 cells. (C) Runx 2 and OSX were tested on the 3 rd day of differentiation, ALP and OCN were tested on the 7 th day of differentiation in MEF cells. "p $<0.05$, *** $\mathrm{p}<0.01$ and ${ }^{* * * *} \mathrm{p}<0.001$.

and MEF cells indicated above for 7 days. The results showed that compared with NC group (BMP9 + NC), exogenous overexpression of miR-155 (BMP9 + miR-155) decreased the expression of OCN and OPN, and downregulated miR-155 group (BMP9 + anti-miR-155) eliminated this inhibitory effect in C2C12 cells (Fig. 4B) and MEF cells (Fig. 5B). The above data demonstrated that inhibition of miR-155 led to BMP signaling that coincided with increased bone formation, strongly suggested that miR-155 suppressed BMP9-induced osteogenic differentiation by repressing BMP signaling.

miR-155 directly targets Runx 2 and BMPR 2 in BMP signaling. To further study the mechanisms of miR-155 inhibited osteogenic differentiation induced by BMP9, we used biological information analysis to find the potential targets of miR-155 in BMP signaling. From the target prediction tools (TargetScan 

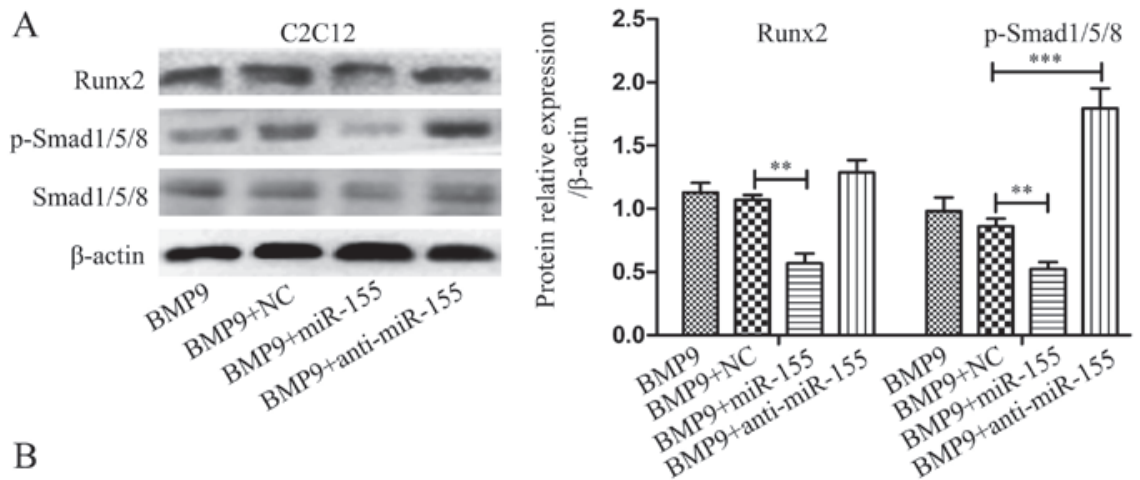

B
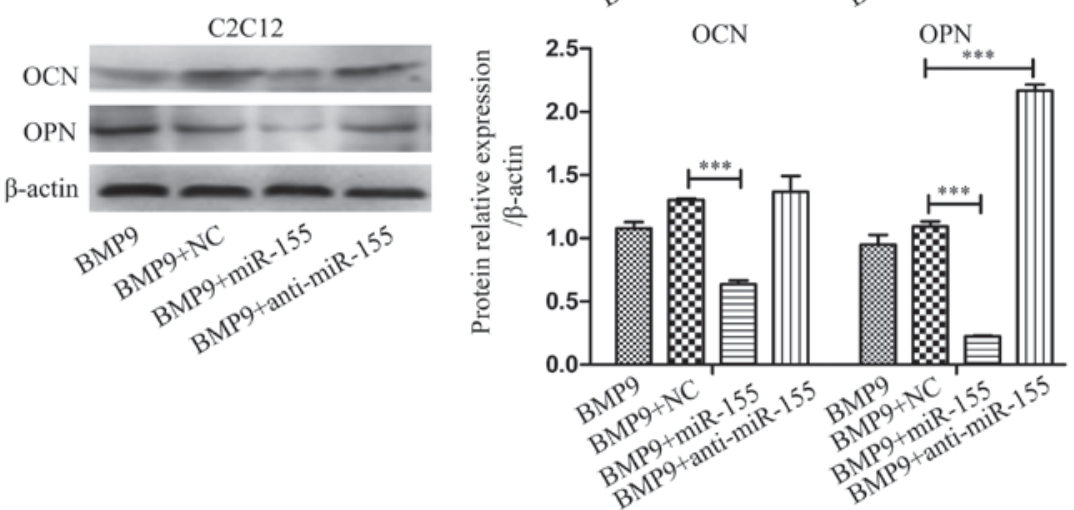

Figure 4. miR-155 acts as an inhibitor of osteogenesis via suppressing BMP signaling during osteogenesis induced by bone morphogenetic protein 9 (BMP9) in $\mathrm{C} 2 \mathrm{C} 12$ cells. (A) After treatment of $\mathrm{C} 2 \mathrm{C} 12$ cells, the expression of $\mathrm{p}$-Smad1/5/8 and runt-related transcription factor 2 (Runx2) were detected by western blotting. (B) The protein expression levels of osteocalcin (OCN) and OPN were measured by western blotting. $\beta$-actin was used as the internal controls, ${ }^{* *} \mathrm{p}<0.01$ and $^{* * * *} \mathrm{p}<0.001$.

A

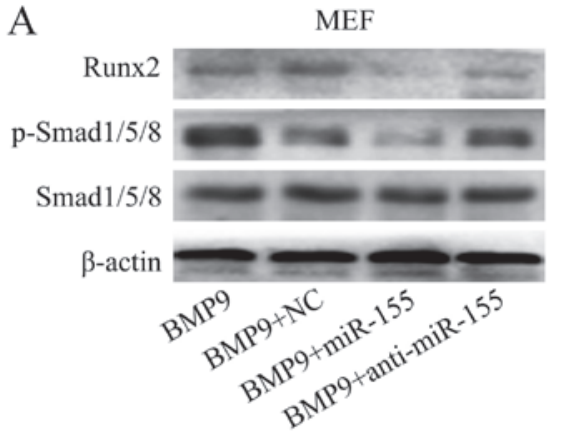

B

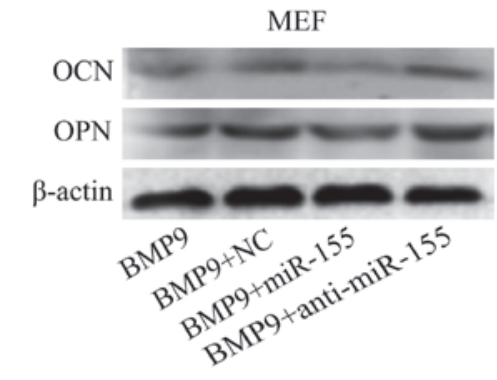

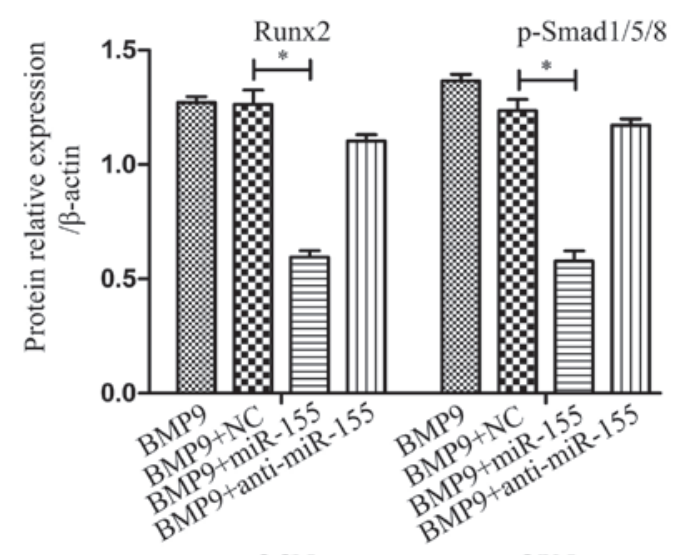

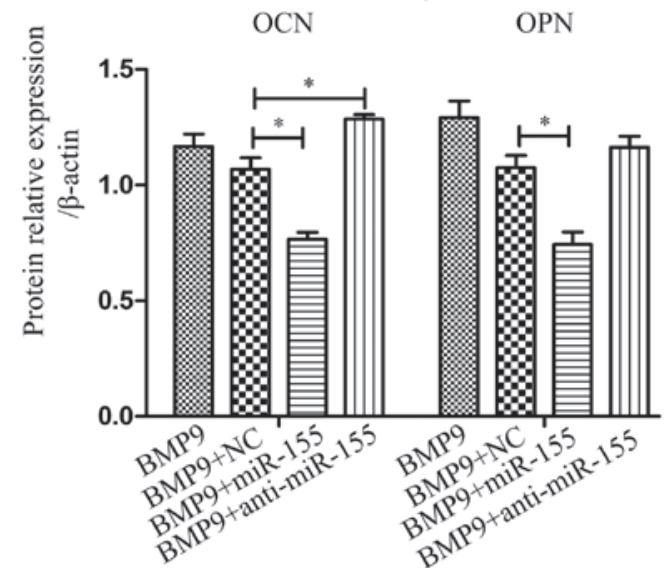

Figure 5. miR-155 acts as an inhibitor of osteogenesis via suppressing BMP signaling during the osteogenesis induced by bone morphogenetic protein 9 (BMP9) in mouse embryonic fibroblasts (MEF) cells. (A) After treatment of MEF cells, the expression of p-Smad1/5/8 and runt-related transcription factor 2 (Runx2) was detected by western blotting. (B) The protein expression levels of osteocalcin (OCN) and osteopontin (OPN) were measured by western blotting. $\beta$-actin was used as the internal controls, ${ }^{\prime} \mathrm{p}<0.05$. 

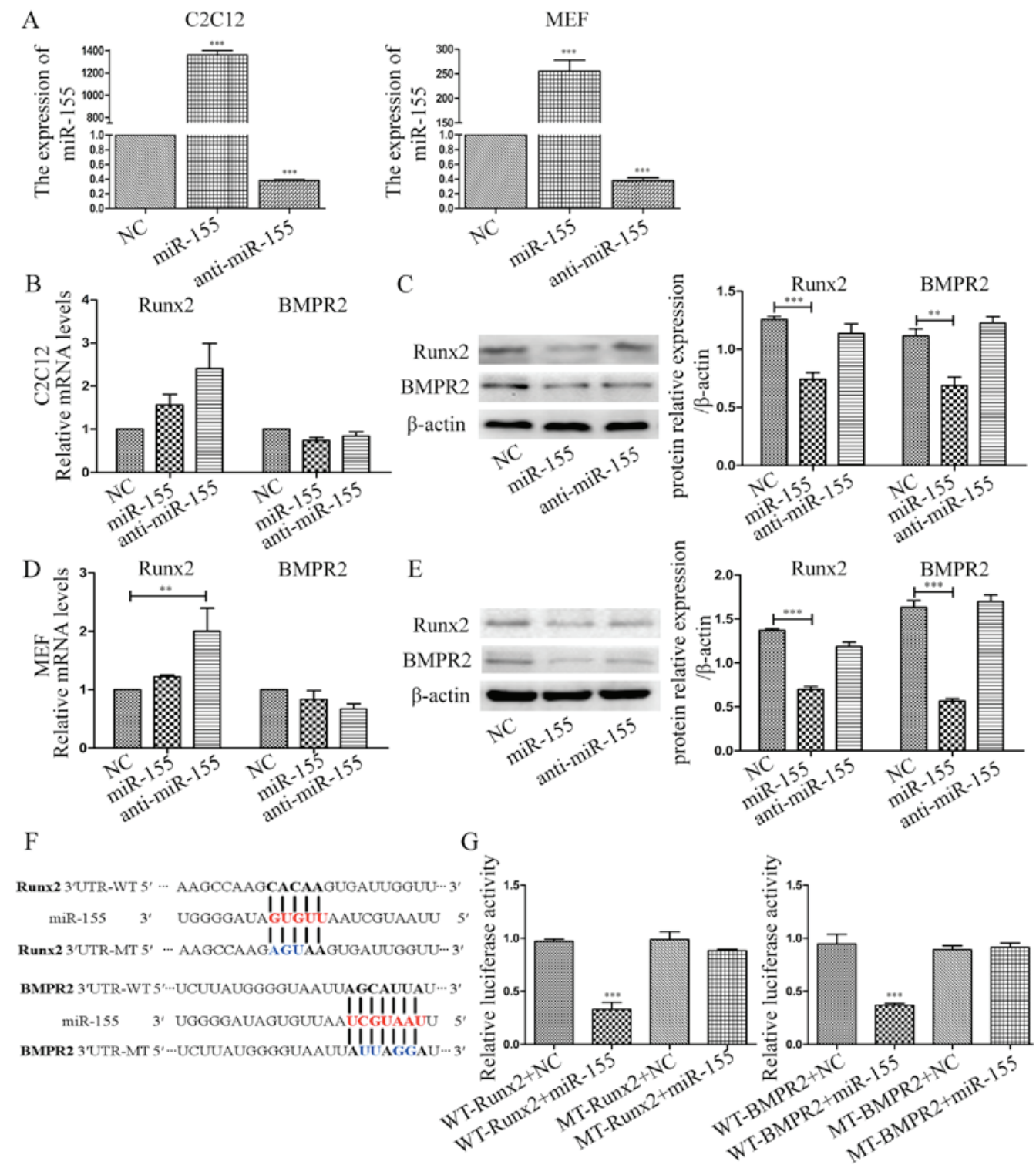

Figure 6. miR-155 directly targets runt-related transcription factor 2 (Runx2) and BMPR2. C2C12 cells and mouse embryonic fibroblasts (MEF) were transfected with NC, miR-155 or anti-miR-155, respectively. (A) Detected the expression of miR-155 by RT-qPCR. (B) Tested the expression of Runx2 and BMPR2 in C2C12 cells by RT-qPCR. (C) The expression of Runx2 and BMPR 2 in $\mathrm{C} 2 \mathrm{C} 12$ cells by western blotting. (D) The expression of Runx2 and BMPR2 in MEF cells by RT-qPCR. (E) The expression of Runx2 and BMPR2 in MEF cells by western blotting. (F) Putative binding sites for miR-155 in the 3'-untranslated region (3'-UTR) of Runx2 and BMPR2 as predicted by TargetScan and PicTar. Mutated nucleotides in mutant Runx2 3'-UTR and BMPR2 3'-UTR seed sequences are represented in blue. (G) Luciferase reporter assays in HEK-293 cells. Cells were co-transfected with WT-Runx2, or MT-Runx2, or WT-BMPR2 or MT-BMPR2 reporter construct or miR-155 overnight. Forty-eight hours post transfection luciferase activities were tested. Results were normalized to the value of cells that transfected pMIR-REPORT only. $\beta$-actin was used as the internal controls for western blotting, ${ }^{* *} \mathrm{p}<0.01$ and ${ }^{* * *} \mathrm{p}<0.001$.

and PicTar), we found the target binding sites of miR-155 on the 3'-UTR of Runx2 and BMPR2, which can complementary bind with miR-155 seed sequence. In order to evaluate whether Runx 2 and BMPR2 are direct targets of miR-155, we measured the mRNA and protein expression levels following the regulation of miR-155 expression by transfecting the MSCs with NC, miR-155, or anti-miR-155. After determining the transfection efficiency (Fig. 6A), we discovered that in overexpressedmiR-155 C2C12 cells, the mRNA expression level of Runx2 and BMPR2 was not significantly altered compared with NC. In anti-miR-155 group, the expression levels had no significant changes as miR-155 group compared with NC (Fig. 6B). While, in MEF cells, the expression of Runx 2 was increased in anti-miR-155 group compared with NC group, the expres- sion level was not significantly altered in miR-155 group. The mRNA expression of BMPR2 had no markedly significant differences in different groups (Fig. 6D). However, compared with $\mathrm{NC}$, the protein expression level of Runx2 and BMPR 2 was decreased in miR-155 group in $\mathrm{C} 2 \mathrm{C} 12$ cells and MEF cells, respectively (Fig. 6C and E). These results demonstrated that miR-155 significantly decreased the protein expression level of Runx2 and BMPR2, but did not markedly altered the mRNA expression levels, as compared with $\mathrm{NC}$, respectively.

To test whether miR-155 directly binds to the 3'-UTR of Runx2 and BMPR2, luciferase reporter assays were performed in HEK-293 cells. We directly designed and synthesized sequences matching miR-155 target sites and cloned these 

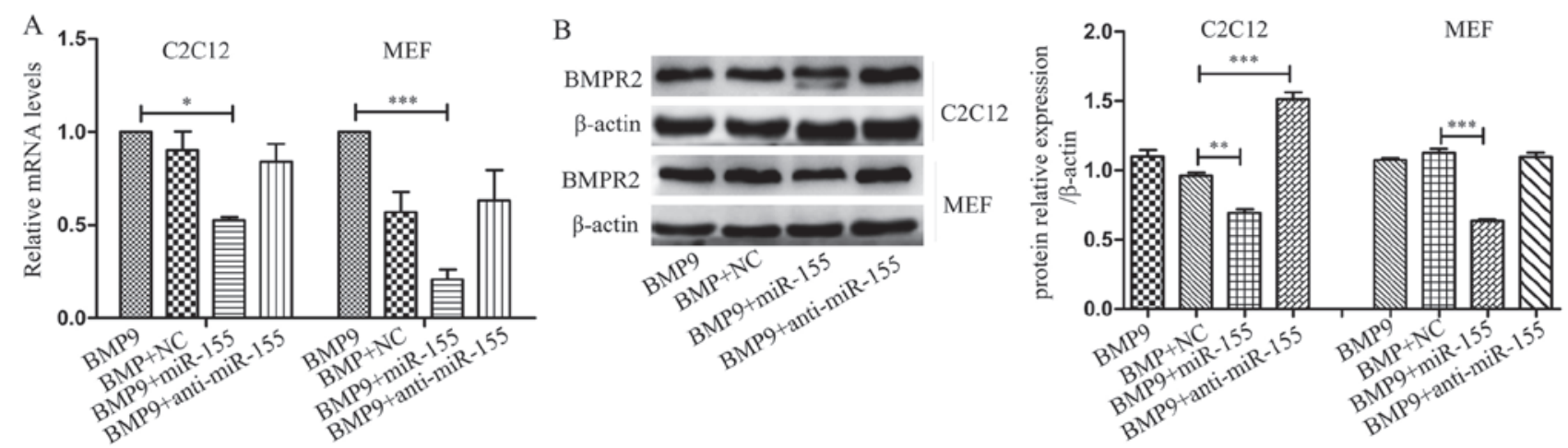

Figure 7. During the osteogenic differentiation induced by bone morphogenetic protein 9 (BMP9), miR-155 decreases the expression of BMPR 2 . (A) The mRNA expression of BMPR2 in C2C12 cells and mouse embryonic fibroblasts (MEF) cells by RT-qPCR. (B) The protein expression of BMPR2 in C2C12 cells and MEF cells by western blot. $\beta$-actin was used as the internal controls, ${ }^{*} \mathrm{p}<0.05,{ }^{* * *} \mathrm{p}<0.01$ and ${ }^{* * * *} \mathrm{p}<0.001$.
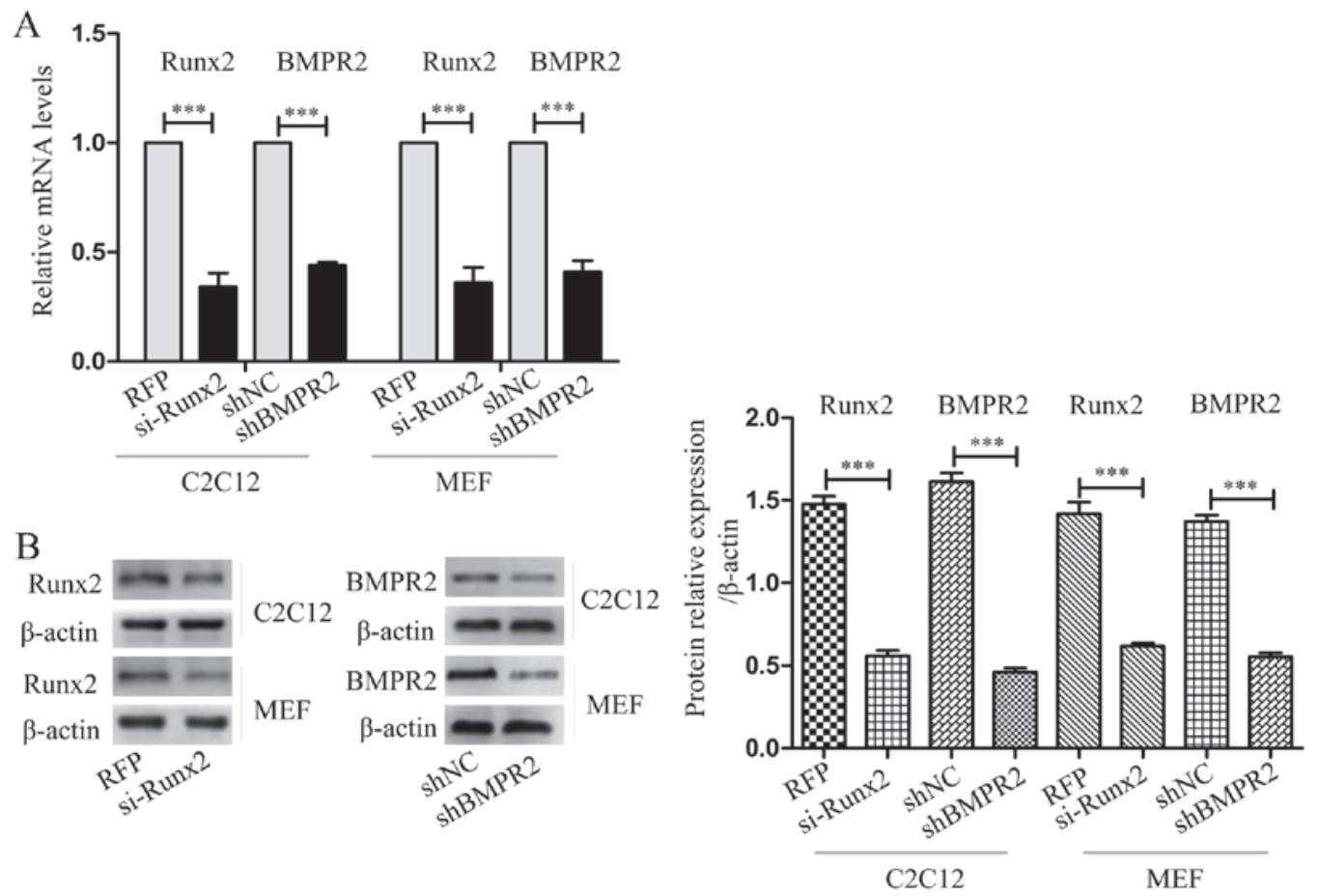

Figure 8. Interference of runt-related transcription factor 2 (Runx2) and BMPR2 in C2C12 cells and mouse embryonic fibroblasts (MEF), respectively. (A) The interference efficiency of si-Runx 2 and shBMPR 2 in $\mathrm{C} 2 \mathrm{C} 12$ cells and mouse embryonic fibroblasts (MEF) after transfection by RT-qPCR. (B) The interference efficiency of si-Runx 2 and shBMPR 2 in $\mathrm{C} 2 \mathrm{C} 12$ cells and MEF cells after transfection by western blotting. $\beta$-actin was used as the internal controls, ${ }^{* * *} \mathrm{p}<0.001$.

wild-type and mutant-type into the multiple cloning sites of pMIR-REPORT luciferase, termed WT-Runx2, MT-Runx2, WT-BMPR2 and MT-BMPR2 (Fig. 6F). Luciferase levels were significantly reduced when cells were co-transfected with WT-Runx2 and miR-155 compared with the cells that co-transfected with WT-Runx2 and NC. However, this difference was abolished when the binding site was mutated suggesting a specific Runx2::miR-155 direct interaction (Fig. 6G). For BMPR2, the result was the same as Runx2, when co-transfected with WT-BMPR2 and miR-155 into HEK-293 cells, luciferase activity was decreased remarkable, mutated binding site could offset this depressant effect demonstrating a specific BMPR2::miR-155 direct interaction (Fig. 6G). In conclusion, the results suggested that Runx2 and BMPR2 are direct targets of miR-155.
miR-155 decreases the the expression of Runx 2 and BMPR 2 in the osteogenic differentiation induced by BMP9. To validate the role of Runx2 and BMPR2 in miR-155 inhibited osteogenic differentiation of MSCs induced by BMP9, we tested the expression changes of Runx2 and BMPR2 under the influence of miR-155 in osteogenesis. In C2C12 cells and MEF cells, in the process of differentiation, compared with NC, miR-155 repressed the expression of Runx2 at mRNA level (Fig. 3B and C) and protein level (Figs. 4A and 5A), and anti-miR-155 reduced the inhibitory effect of miR-155 on the expression of Runx 2 and BMPR2. In C2C12 and MEF cells, miR-155 had no obvious influence at the expression of mRNA level, compared with NC during the differentiation (Fig. 7A). However, it reduced the protein expression level of BMPR2, and anti-miR-155 promoted the protein level in $\mathrm{C} 2 \mathrm{C} 12$ cells compared with NC (Fig. 7B). 

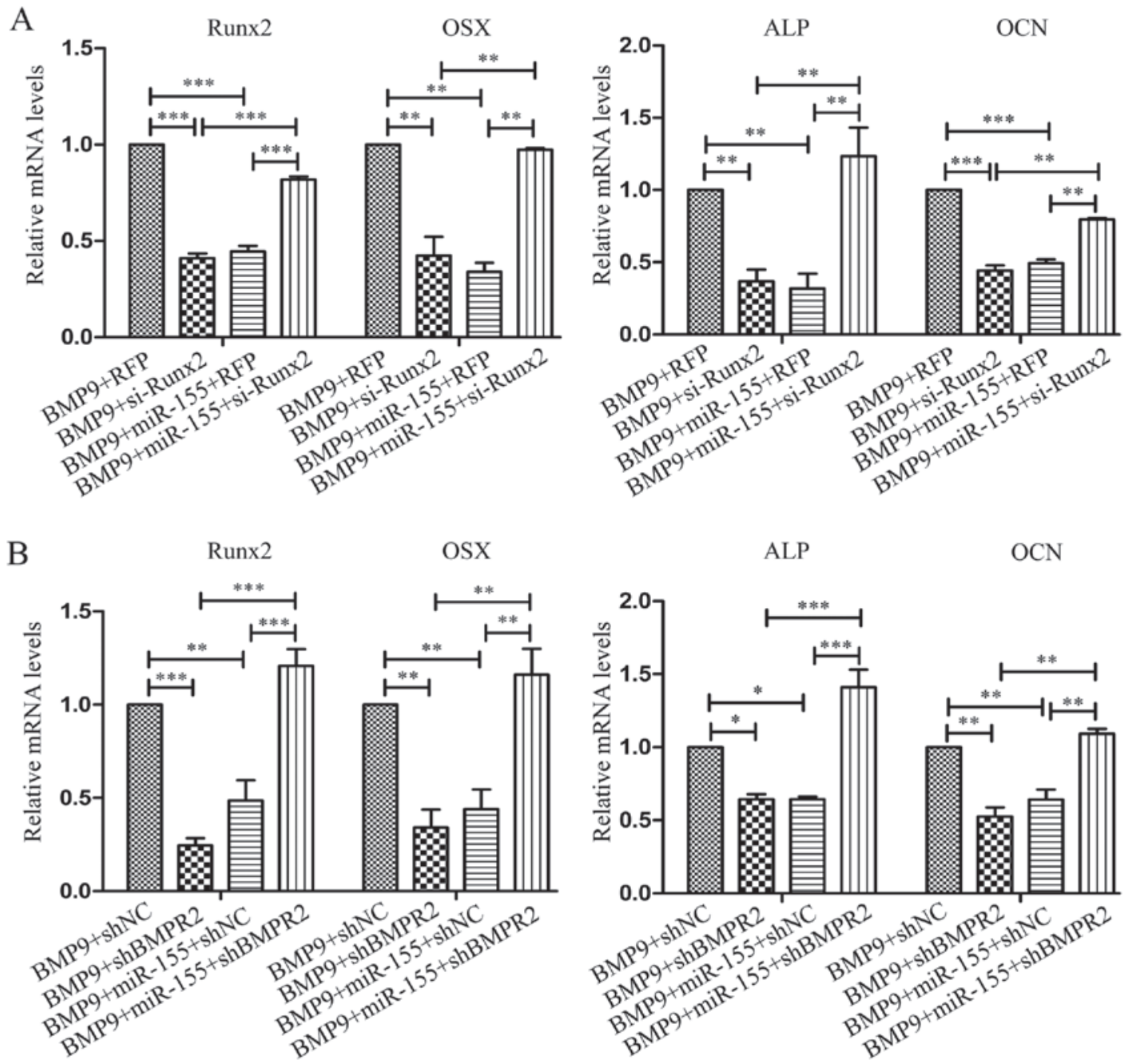

Figure 9. Knockdown of runt-related transcription factor 2 (Runx2) and BMPR2 separately reduced the effect of miR-155 in C2C12 cells. (A and B) C2C12 cells were co-transfected with RFP, or si-Runx2, or shNC, or shBMPR2, or miR-155, then induced osteogenic differentiation by bone morphogenetic protein 9 (BMP9) for 3 days to test the expression of Runx2, osterix (OSX), and induced for 7 days to test the expression of alkaline phosphatase (ALP), osteocalcin (OCN) used RT-qPCR. ${ }^{*} \mathrm{p}<0.05,{ }^{* *} \mathrm{p}<0.01$ and $^{* * * *} \mathrm{p}<0.001$.

Knockdown of Runx2 and BMPR2 reduce the inhibitory effect of miR-155 on the osteogenic differentiation induced by BMP9. To further verify the association between miR-155 and its target genes, Runx2, and BMPR2, we blocked the expression of Runx2 by si-Runx2, and blocked the expression of BMPR2 by shBMPR2. Through blocking the target genes of miR-155, the effect of miR-155 on osteogenesis of MSCs was evaluated, to speculate on the function of the target genes of miR-155. After transfecting si-Runx2, shBMPR2 or their controls into $\mathrm{C} 2 \mathrm{C} 12$ cells and MEF cells, respectively, used RT-qPCR to examine the interfering efficiency, data showed that compared with the control groups, the expression of Runx2 and BMPR2 was significantly decreased (Fig. 8A). The results of western blot analysis shown that the protein expression of Runx 2 and BMPR2 was significantly reduced compared with their control group, respectively (Fig. 8B).

In $\mathrm{C} 2 \mathrm{C} 12$ cells, the expression of Runx2, OSX, ALP and OCN were distinctly decreased when transfected with si-Runx2 (BMP9 + si-Runx2) compared with RFP (BMP9 + RFP); and in co-transfection with miR-155 and si-Runx2 group (BMP9 + miR-155 + si-Runx2), the expression of these four bone-related genes were significantly increased compared with co-transfection with miR-155 and RFP group (BMP9 + miR-155 + RFP) (Fig. 9A). In the process of osteogenesis, after transfecting shBMPR2 (BMP9 + shBMPR2), the expression of Runx2, OSX, ALP and $\mathrm{OCN}$ were markedly decreased compared with control group (BMP9 + shNC); in co-transfected miR-155 and sh-BMPR2 group (BMP9 + miR-155 + sh-BMPR2), the expression of these four bone differentiation related genes was notably increased compared with co-transfected with miR-155 and shNC group (BMP9 + miR-155 + shNC) (Fig. 9B). The results obtained from MEF cells coincided with the results of $\mathrm{C} 2 \mathrm{C} 12$ cells (Fig. 10), when co-transfected with miR-155 and si-Runx 2 or sh-BMPR2, the expression of Runx2, OSX, ALP and OCN was remarkably increased compared with co-transfected with miR-155 and RFP or shNC, respectively. These data indicated that knockdown of Runx2 and BMPR2, respectively, the effect of miR-155 on the osteogenic differentiation induced by BMP 9 was reduced. In conclusion, miR-155 inhibited osteogenic differentiation induced by BMP9 may be through repression of the expression of its target genes-Runx2 and BMPR2, which are, at least partially, quite important components in BMP signaling. 

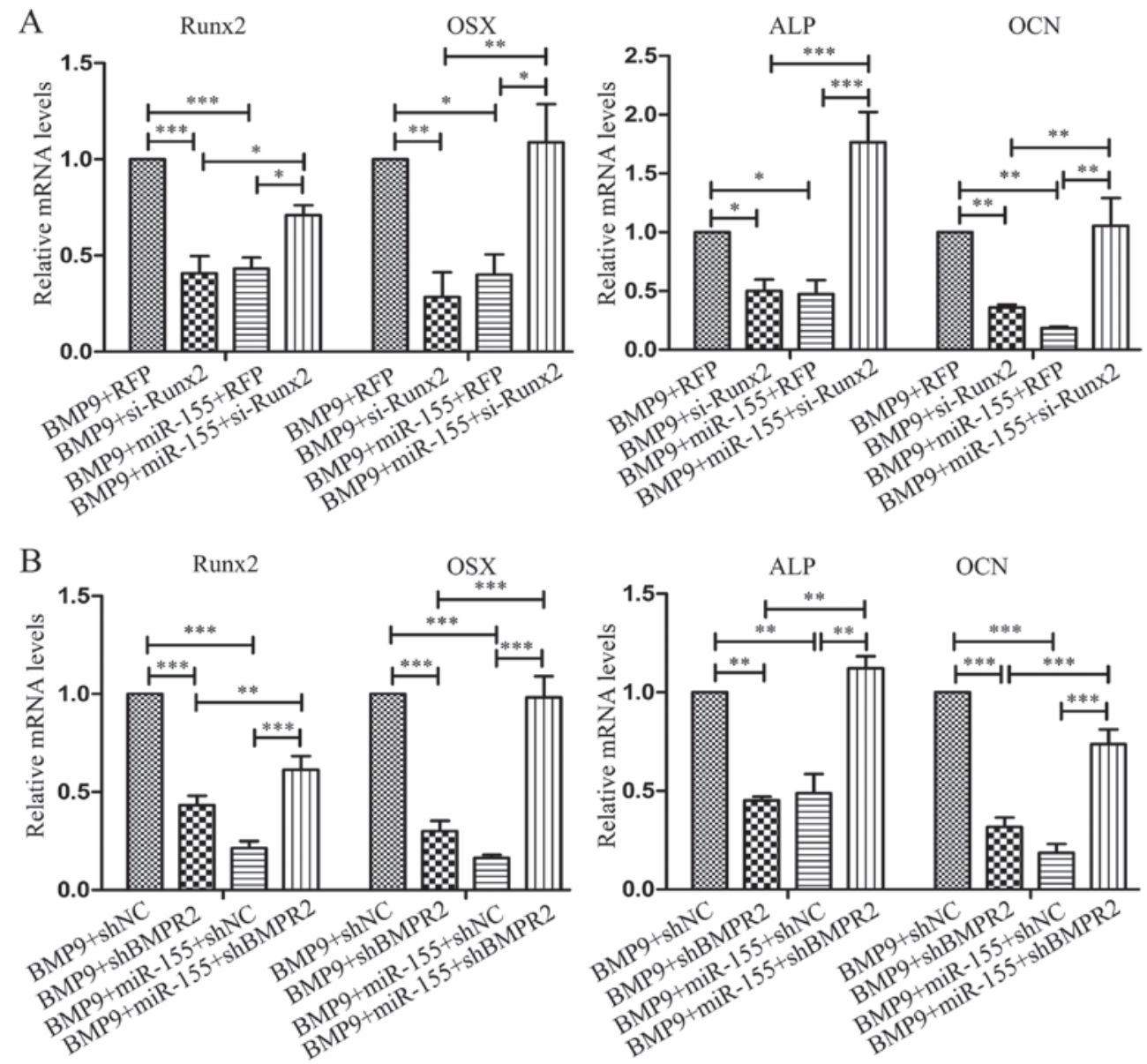

Figure 10. Knockdown runt-related transcription factor 2 (Runx2) and BMPR2 separately reduced the effect of miR-155 in mouse embryonic fibroblasts (MEF) cells. (A and B) MEF cells were co-transfected with RFP or si-Runx 2 or shNC or shBMPR2 and miR-155, then induced osteogenic differentiation by BMP9 for 3 days to test the expression of Runx2, osterix (OSX), was induced for 7 days to test the expression of alkaline phosphatase (ALP) and osteocalcin (OCN) by RT-qPCR. ${ }^{*} \mathrm{p}<0.05,{ }^{* *} \mathrm{p}<0.01$ and ${ }^{* * *} \mathrm{p}<0.001$.

Effect of miR-155 on in vivo ectopic bone formation of BMP9. To determine the consequences of miR-155 on osteogenic differentiation induced by BMP9 in vivo, we performed $\mu \mathrm{CT}$ analysis of heterotopic bones from 10-week-old female nude mice. The microarchitecture of bones were in accordance with data from cell culture experiments, $\mu \mathrm{CT}$ images of ectopic bones showed decreased trabecular bone volume in BMP9 + agomiR-155 group compared to BMP9 + NC group, while in BMP9 + antagomiR-155 group, the trabecular bone volume was increased compared with BMP9 + NC group (Fig. 11A). In $\mu \mathrm{CT}$ analyses, BMP9 + agomiR-155 group exhibited significant decrease in BMD, BV/TV and Tb.Th, but a significant increase in Tb.SP, there were no variances in Tb.N compared with BMP9 + NC group, respectively. On the contrary, antago-miR-155 elevated BV/TV and Tb.Th of the bones consequently, and had no effect on Tb.N, Tb.SP and BMD in the ectopic bone induction of BMP9 as well (Fig. 11B). After the $\mu \mathrm{CT}$ scan, all specimens were subjected to a histological study involving H\&E staining and Masson's trichrome staining. In histological analyses, the results correspond to the results that we gained from cell culture in vitro. agomiR-155 inhibited the osteogenic differentiation of MEF cells induced by BMP9 in vivo, decreased trabecular bone mass, whereas antagomiR-155 reversed this phenomenon (Fig. 11C). The results of Masson's trichrome staining were very similar to the
H\&E staining in all the groups, showing that the chondrocytes were stained in BMP9 + antagomir-155 group more, but in BMP9 + agomiR-155 group, the staining was lighter compared with BMP9 + NC, respectively (Fig. 11D). Given the above, in vivo, agomiR-155 depressed the ectopic bone formation of MEF cells induced by BMP9, antagomiR-155 facilitated ectopic bone formation, conversely.

\section{Discussion}

Osteoblasts are a class of bone-forming cells that originate from bone marrow stromal (skeletal or mesenchymal) stem cells and are responsible for bone growth during development and bone formation during remodeling of the post-natal skeleton. Previous studies identified BMP9 as one of the most powerful osteogenic BMPs, both in vitro and in vivo $(2,4,9,29)$. As one of the most osteogenic BMPs, BMP9 can induce MSCs to differentiate into osteoblasts (46-48). But the specific mechanisms of BMP9 osteoinduction are not very clear, it is necessary to investigate the complete regulatory mechanisms of BMP9-induced osteogenic differentiation.

In this study, during the early stage of BMP9-induced osteogenic differentiation of MSCs, the expression of miR-155 presented early increased and later decreased trend, indicated that there are some relationship between miR-155 and BMP9. 
A

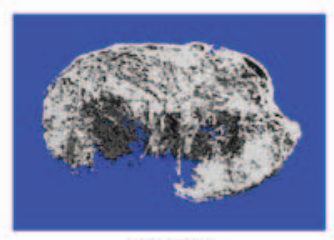

BMP9

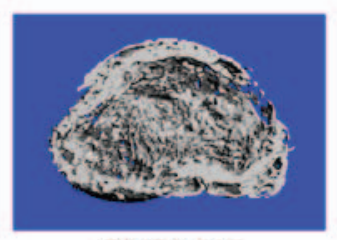

BMP9+NC

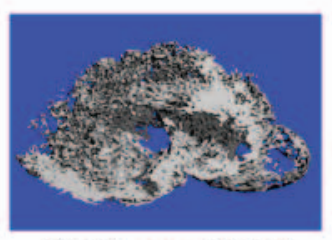

BMP9+agomiR-155

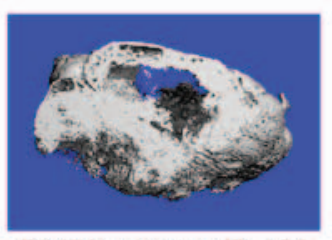

BMP9+antagomiR-155
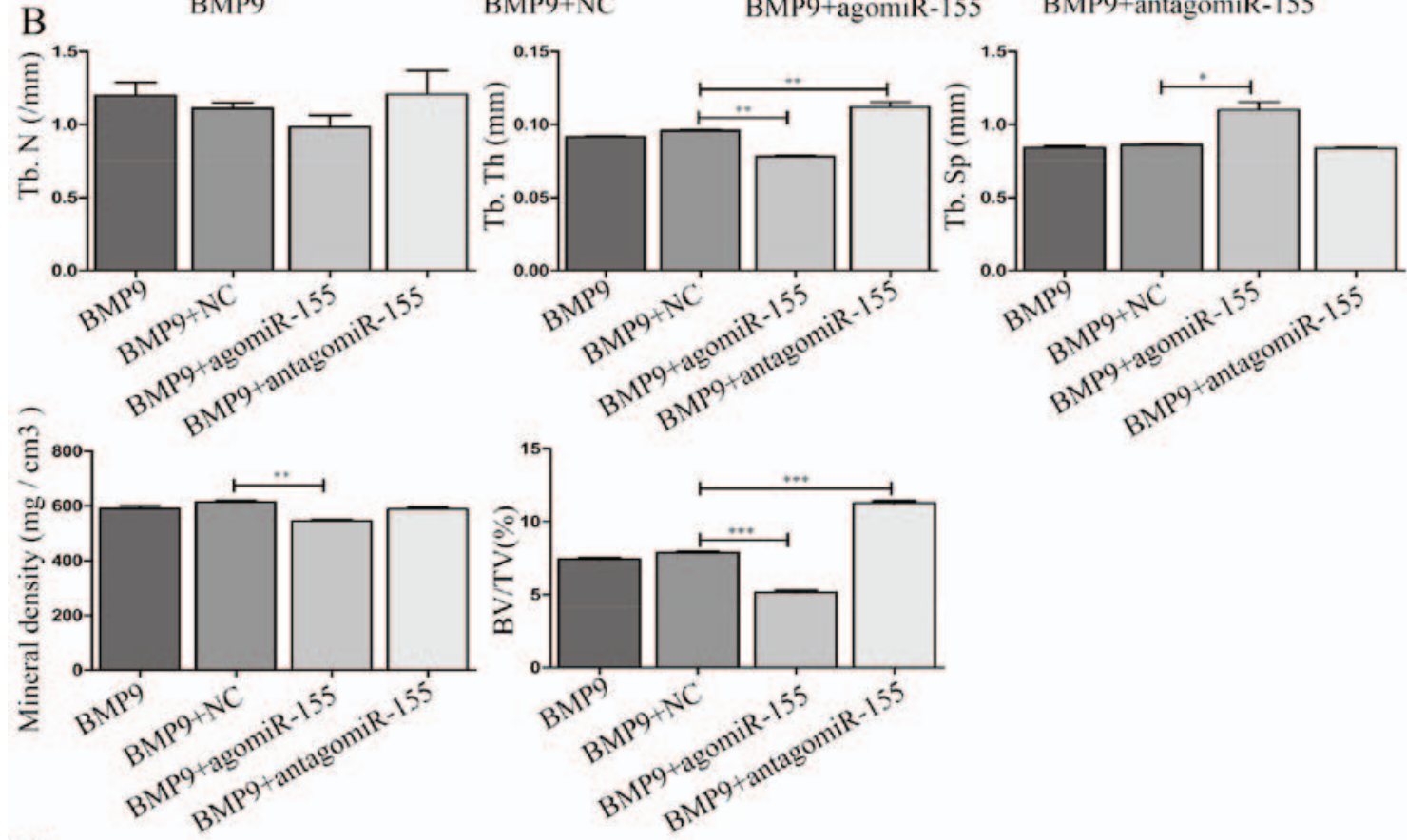

C

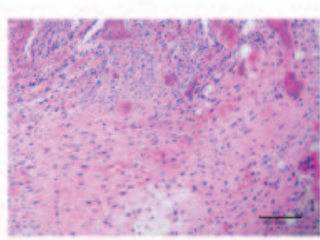

BMP9

D

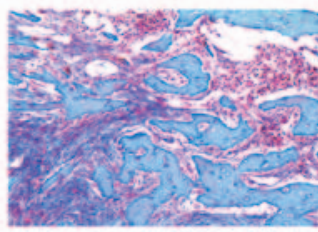

BMP9

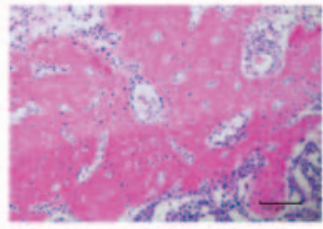

BMP9+NC

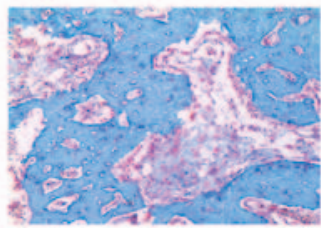

$\mathrm{BMP} 9+\mathrm{NC}$

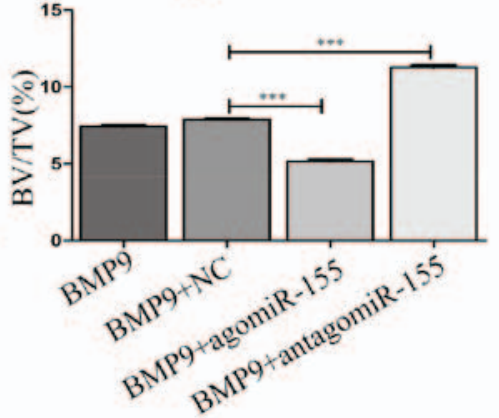

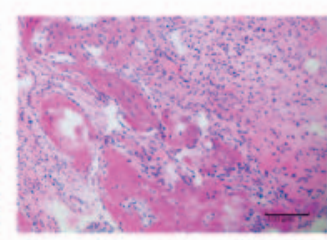

BMP9+agomiR-155

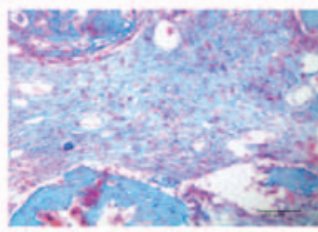

BMP9+agomiR-155

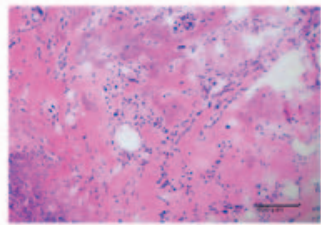

BMP9+antagomiR-155

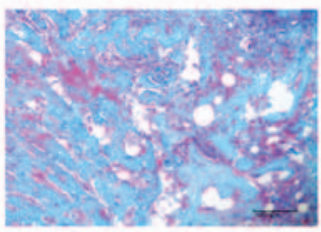

BMP9+antagomiR-155

Figure 11. miR-155 repressed ectopic bone formation induced by bone morphogenetic protein 9 (BMP9) in vivo. Ectopic bones were harvested 6 weeks after treated-mouse embryonic fibroblasts (MEF) cells were injected subcutaneously in 4-week-old female nude mice. (A) $\mu \mathrm{CT}$ with representative image of the ectopic bones. Scale bar, $1 \mathrm{~mm}$. (B) $\mu \mathrm{CT}$ quantification of the ectopic bones from 10 -week-old female nude mice, which were injected with transfected-MEF cells at 4 weeks. Parameters include: trabecular number, thickness, separation, volume and BMD. $\mathrm{n}=3,{ }^{*} \mathrm{p}<0.05,{ }^{* *} \mathrm{p}<0.01$ and ${ }^{* * * *} \mathrm{p}<0.001$. (C) Histological images of 6-week ectopic bones from each group upon H\&E staining. Scale bars, $100 \mu \mathrm{m}$. (D) Histological images of 6-week ectopic bones from each group upon Masson's trichrome staining. Scale bars, $100 \mu \mathrm{m}$.

A one study indicated that miR-155 could modulate the inhibitory effect of TNF- $\alpha$ on BMP-2-induced osteogenic differentiation in MC3T3-E1 cells (43), suggested potential relationships among them. Based on the staining experiments, we speculated miR-155 may be a negative regulator in osteogenic differentiation. The results of in vitro supported our hypothesis suggesting that miR-155 inhibits the osteogenic differentiation of MSCs induced by BMP9 may be via suppressing Smad/BMP signaling activity and repressing the expression of Runx2 and BMPR2. Although, in some cases, anti-miR-155 had a modest effect on the expression of bone-related genes compared with $\mathrm{NC}$, suggested that antimiR-155 could reverse the inhibitory effect of miR-155 on the osteogenic differentiation of MSCs induced by BMP9, sufficiently. In vivo, agomiR-155 repressed the ectopic bone formation, providing more believable evidence to prove our hypothesis.

Early studies revealed that canonical Smad-dependent signaling pathway and non-canonical Smad-independent signaling (p38 MAPKs) pathway are involved in BMP9-induced osteogenic differentiation of MSCs. MAPKs are a group of well-described serine/threonine-specific protein kinases gener- 
ally expressed in all cell types $(9,29,48)$. At least four subfamilies of mammalian MAPKs have been identified, including ERK1/2, ERK5 (also known as MAPK7 or BMK1), the Jun aminoterminal kinases (JNKs) and p38 MAPKs. ERK1/2, JNKs, and p38 are involved in BMP9-induced osteogenic differentiation of MSCs $(44,45,49,50)$. Whether miR-155 influence the noncanonical Smad-independent signaling (p38 mitogen-activated protein kinase, MAPK) pathway has not been investigated, to study the mechanisms further, we can focus on this aspect. As Runx 2 and BMPR2 were verified as the target genes of miR-155 in our study, they were interfered by siRNA, or shRNA to block the targets of miR-155. The results showed that miR-155 failed to inhibit osteogenic differentiation, suggesting miR-155 may play negative regulatory roles in BMP9-induced osteogenic differentiation through repressed expression of Runx2 and BMPR2. More regulatory factors and the corresponding mechanisms in the process of BMP9-induced osteogenic differentiation need to be explored. Finding more target genes of miR-155 in BMP signaling is beneficial to understand the mechanism of action of miR-155, promote the clinic application of BMP9 finally.

\section{Acknowledgements}

The authors would like to thank T.C. He (Medical Center, the University of Chicago) for his kind provision of the recombinant adenovirus (BMP9 and si-Runx2).

\section{Funding}

This study was supported by the National Natural Science Foundation of China (NSFC 81672103), the National Natural Science Foundation of China (NSFC 31200971), the National Ministry of Education Foundation of China (20115503110009) and the Program of the Ministry of Science and Technology of Yu-zhong District, CQ (20130136).

\section{Availability of data and material}

All data generated or analyzed during this study are included in this published article.

\section{Authors' contributions}

HL designed the experiments, controlled the progress of the research, and was a major contributor in writing the manuscript. LZ, TY, YZ, YG, MF, YL, YS, WL and SC critically revised the manuscript for important intellectual content. LA was involved in animal experiments of the nude mice preliminary treatment. YW and QS analyzed the data and revised the manuscript. All authors read and approved the final manuscript.

\section{Ethics approval and consent to participate}

All animal experiments were approved by the Institutional Animal Care and Use Committee (IACUC) of Chongqing Medical University (reference no. 2016009).

\section{Consent for publication}

Not applicable.

\section{Competing interests}

The authors declare that they have no competing interests.

\section{References}

1. Luu HH, Song WX, Luo X, Manning D, Luo J, Deng ZL, Sharff KA, Montag AG, Haydon RC and He TC: Distinct roles of bone morphogenetic proteins in osteogenic differentiation of mesenchymal stem cells. J Orthop Res 25: 665-677, 2007.

2. Beederman M, Lamplot JD, Nan G, Wang J, Liu X, Yin L, Li R, Shui W, Zhang H, Kim SH, et al: BMP signaling in mesenchymal stem cell differentiation and bone formation. J Biomed Sci Eng 6: 32-52, 2013.

3. Bidart M, Ricard N, Levet S, Samson M, Mallet C, David L, Subileau M, Tillet E, Feige JJ and Bailly S: BMP9 is produced by hepatocytes and circulates mainly in an active mature form complexed to its prodomain. Cell Mol Life Sci 69: 313-324, 2012.

4. Brown MA, Zhao Q, Baker KA, Naik C, Chen C, Pukac L, Singh M, Tsareva T, Parice Y, Mahoney A, et al: Crystal structure of BMP-9 and functional interactions with pro-region and receptors. J Biol Chem 280: 25111-25118, 2005.

5. Wang RN, Green J, Wang Z, Deng Y, Qiao M, Peabody M, Zhang Q, Ye J, Yan Z, Denduluri S, et al: Bone Morphogenetic Protein (BMP) signaling in development and human diseases. Genes Dis 1: 87-105, 2014.

6. Yan F, Luo S, Jiao Y, Deng Y, Du X, Huang R, Wang Q and Chen W: Molecular characterization of the BMP7 gene and its potential role in shell formation in Pinctada martensii. Int J Mol Sci 15: 21215-21228, 2014.

7. Miyazono K, Kamiya Y and Morikawa M: Bone morphogenetic protein receptors and signal transduction. J Biochem 147: 35-51, 2010.

8. Rahman MS, Akhtar N, Jamil HM, Banik RS and Asaduzzaman SM: TGF- $\beta$ /BMP signaling and other molecular events: Regulation of osteoblastogenesis and bone formation. Bone Res 3: 15005, 2015.

9. Chen L, Jiang W, Huang J, He BC, Zuo GW, Zhang W, Luo Q Shi Q, Zhang BQ, Wagner ER, et al: Insulin-like growth factor 2 (IGF-2) potentiates BMP-9-induced osteogenic differentiation and bone formation. J Bone Miner Res 25: 2447-2459, 2010.

10. Kang Q, Song WX, Luo Q, Tang N, Luo J, Luo X, Chen J, Bi Y, He BC, Park JK, et al: A comprehensive analysis of the dual roles of BMPs in regulating adipogenic and osteogenic differentiation of mesenchymal progenitor cells. Stem Cells Dev 18: 545-559, 2009.

11. Hua Z, Lv Q, Ye W, Wong CK, Cai G, Gu D, Ji Y, Zhao C, Wang J, Yang BB, et al: MiRNA-directed regulation of VEGF and other angiogenic factors under hypoxia. PLoS One 1: e116, 2006.

12. Wang X, Guo B, Li Q, Peng J, Yang Z, Wang A, Li D, Hou Z, Lv K, Kan G, et al: miR-214 targets ATF4 to inhibit bone formation. Nat Med 19: 93-100, 2013.

13. Sevli S, Uzumcu A, Solak M, Ittmann M and Ozen M: The function of microRNAs, small but potent molecules, in human prostate cancer. Prostate Cancer Prostatic Dis 13: 208-217, 2010.

14. Alshalalfa M: MicroRNA response elements-mediated miRNA-miRNA interactions in prostate cancer. Adv Bioinforma 2012: 839837, 2012.

15. Eskildsen T, Taipaleenmäki H, Stenvang J, Abdallah BM, Ditzel N, Nossent AY, Bak M, Kauppinen S and Kassem M: MicroRNA-138 regulates osteogenic differentiation of human stromal (mesenchymal) stem cells in vivo. Proc Natl Acad Sci USA 108: 6139-6144, 2011.

16. Itoh T, Nozawa Y and Akao Y: MicroRNA-141 and -200a are involved in bone morphogenetic protein-2-induced mouse preosteoblast differentiation by targeting distal-less homeobox 5 . J Biol Chem 284: 19272-19279, 2009.

17. Kureel J, Dixit M, Tyagi AM, Mansoori MN, Srivastava K, Raghuvanshi A, Maurya R, Trivedi R, Goel A and Singh D: miR-542-3p suppresses osteoblast cell proliferation and differentiation, targets BMP-7 signaling and inhibits bone formation. Cell Death Dis 5: e1050, 2014.

18. Luo W, Nie Q and Zhang X: MicroRNAs involved in skeletal muscle differentiation. J Genet Genomics 40: 107-116, 2013.

19. Qi R, Long D, Wang J, Wang Q, Huang X, Cao C, Gao G and Huang J: MicroRNA-199a targets the fatty acid transport protein 1 gene and inhibits the adipogenic trans-differentiation of C2C12 myoblasts. Cell Physiol Biochem 39: 1087-1097, 2016. 
20. Yu JY, Chung KH, Deo M, Thompson RC and Turner DL: MicroRNA miR-124 regulates neurite outgrowth during neuronal differentiation. Exp Cell Res 314: 2618-2633, 2008.

21. Zhang J, Du YY, Lin YF, Chen YT, Yang L, Wang HJ and Ma D: The cell growth suppressor, mir-126, targets IRS-1. Biochem Biophys Res Commun 377: 136-140, 2008.

22. Xu B, Tao T, Wang Y, Fang F, Huang Y, Chen S, Zhu W and Chen M: hsa-miR-135a-1 inhibits prostate cancer cell growth and migration by targeting EGFR. Tumour Biol 37: 14141-14151, 2016.

23. Shin S, Moon KC, Park KU and Ha E: MicroRNA-513a-5p mediates TNF- $\alpha$ and LPS induced apoptosis via downregulation of X-linked inhibitor of apoptotic protein in endothelial cells. Biochimie 94: 1431-1436, 2012.

24. Esau C, Kang X, Peralta E, Hanson E, Marcusson EG, Ravichandran LV, Sun Y, Koo S, Perera RJ, Jain R, et al: MicroRNA-143 regulates adipocyte differentiation. J Biol Chem 279: 52361-52365, 2004.

25. Huang J, Zhao L, Xing L and Chen D: MicroRNA-204 regulates Runx 2 protein expression and mesenchymal progenitor cell differentiation. Stem Cells 28: 357-364, 2010.

26. Li Z, Hassan MQ, Volinia S, van Wijnen AJ, Stein JL, Croce CM, Lian JB and Stein GS: A microRNA signature for a BMP2induced osteoblast lineage commitment program. Proc Natl Acad Sci USA 105: 13906-13911, 2008.

27. Mizuno Y, Yagi K, Tokuzawa Y, Kanesaki-Yatsuka Y, Suda T, Katagiri T, Fukuda T, Maruyama M, Okuda A, Amemiya T, et al: miR-125b inhibits osteoblastic differentiation by downregulation of cell proliferation. Biochem Biophys Res Commun 368: 267-272, 2008.

28. Sato MM, Nashimoto M, Katagiri T, Yawaka Y and Tamura M: Bone morphogenetic protein-2 downregulates miR-206 expression by blocking its maturation process. Biochem Biophys Res Commun 383: 125-129, 2009.

29. Zhang W, Zhang L, Zhou Y, Ji X, Liu J, Liu D, Yin P, Peng Y, Hao M, Zhang L, et al: Synergistic effects of BMP9 and miR548d-5p on promoting osteogenic differentiation of mesenchymal stem cells. BioMed Res Int 2015: 309747, 2015.

30. Zhang Y, Xie RL, Croce CM, Stein JL, Lian JB, van Wijnen AJ and Stein GS: A program of microRNAs controls osteogenic lineage progression by targeting transcription factor Runx2. Proc Natl Acad Sci USA 108: 9863-9868, 2011.

31. Li Z, Hassan MQ, Jafferji M, Aqeilan RI, Garzon R, Croce CM, van Wijnen AJ, Stein JL, Stein GS and Lian JB: Biological functions of miR-29b contribute to positive regulation of osteoblast differentiation. J Biol Chem 284: 15676-15684, 2009.

32. Vimalraj S, Partridge NC and Selvamurugan N: A positive role of microRNA-15b on regulation of osteoblast differentiation. J Cell Physiol 229: 1236-1244, 2014

33. Elton TS, Selemon H, Elton SM and Parinandi NL: Regulation of the MIR155 host gene in physiological and pathological processes. Gene 532: 1-12, 2013.

34. Kluiver J, Poppema S, de Jong D, Blokzij1 T, Harms G, Jacobs S, Kroesen BJ and van den Berg A: BIC and miR-155 are highly expressed in Hodgkin, primary mediastinal and diffuse large $\mathrm{B}$ cell lymphomas. J Pathol 207: 243-249, 2005.

35. Lawrie $\mathrm{CH}$ : MicroRNAs and lymphomagenesis: A functional review. Br J Haematol 160: 571-581, 2013.

36. Wang M, Tan LP, Dijkstra MK, van Lom K, Robertus JL, Harms G, Blokzijl T, Kooistra K, van T'veer MB, Rosati S, et al miRNA analysis in B-cell chronic lymphocytic leukaemia: Proliferation centres characterized by low miR-150 and high BIC/miR-155 expression. J Pathol 215: 13-20, 2008.
37. Faraoni I, Antonetti FR, Cardone J and Bonmassar E: miR-155 gene: A typical multifunctional microRNA. Biochim Biophys Acta 1792: 497-505, 2009.

38. Jiang S, Zhang HW, Lu MH, He XH, Li Y, Gu H, Liu MF and Wang ED: MicroRNA-155 functions as an OncomiR in breast cancer by targeting the suppressor of cytokine signaling 1 gene. Cancer Res 70: 3119-3127, 2010.

39. Liu J, Mao Q, Liu Y, Hao X, Zhang S and Zhang J: Analysis of miR-205 and miR-155 expression in the blood of breast cancer patients. Chin J Cancer Res 25: 46-54, 2013.

40. Vigorito E, Perks KL, Abreu-Goodger C, Bunting S, Xiang Z, Kohlhaas S, Das PP, Miska EA, Rodriguez A, Bradley A, et al: microRNA-155 regulates the generation of immunoglobulin class-switched plasma cells. Immunity 27: 847-859, 2007.

41. Yanaihara N, Caplen N, Bowman E, Seike M, Kumamoto K, Yi M, Stephens RM, Okamoto A, Yokota J, Tanaka T, et al: Unique microRNA molecular profiles in lung cancer diagnosis and prognosis. Cancer Cell 9: 189-198, 2006.

42. Chen C, Tang Z, Song Q, Yang M, Shi Q and Weng Y: Downregulated microRNA-23b promotes BMP9-mediated osteogenesis in $\mathrm{C} 2 \mathrm{C} 12$ myoblast cells by targeting Runx2. Mol Med Rep 13: 2492-2498, 2016.

43. Wu T, Xie M, Wang X, Jiang X, Li J and Huang H: miR-155 modulates TNF- $\alpha$-inhibited osteogenic differentiation by targeting SOCS1 expression. Bone 51: 498-505, 2012.

44. Zhao Y, Song T, Wang W, Wang J, He J, Wu N, Tang M, He B and Luo J: P38 and ERK1/2 MAPKs act in opposition to regulate BMP9-induced osteogenic differentiation of mesenchymal progenitor cells. PLoS One 7: e43383, 2012

45. Miyazono K, Maeda S and Imamura T: BMP receptor signaling: Transcriptional targets, regulation of signals, and signaling cross-talk. Cytokine Growth Factor Rev 16: 251-263, 2005.

46. Chen L, Zou X, Zhang R-X, Pi CJ, Wu N, Yin LJ and Deng ZL: IGF1 potentiates BMP9-induced osteogenic differentiation in mesenchymal stem cells through the enhancement of BMP/Smad signaling. BMB Rep 49: 122-127, 2016.

47. Li R, Yan Z, Ye J, Huang H, Wang Z, Wei Q, Wang J, Zhao L, Lu S, Wang X, et al: The prodomain-containing BMP9 produced from a stable line effectively regulates the differentiation of mesenchymal stem cells. Int J Med Sci 13: 8-18, 2016.

48. Peng Y, Kang Q, Cheng H, Li X, Sun MH, Jiang W, Luu HH, Park JY, Haydon RC and He TC: Transcriptional characterization of bone morphogenetic proteins (BMPs)-mediated osteogenic signaling. J Cell Biochem 90: 1149-1165, 2003.

49. Xu DJ, Zhao YZ, Wang J, He JW, Weng YG and Luo JY: Smads, p38 and ERK1/2 are involved in BMP9-induced osteogenic differentiation of C3H10T1/2 mesenchymal stem cells. BMB Rep 45: 247-252, 2012.

50. Zhao YF, Xu J, Wang WJ, Wang J, He JW, Li L, Dong Q, Xiao Y, Duan XL, Yang X, et al: Activation of JNKs is essential for BMP9-induced osteogenic differentiation of mesenchymal stem cells. BMB Rep 46: 422-427, 2013.

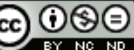

This work is licensed under a Creative Commons Attribution-NonCommercial-NoDerivatives 4.0 International (CC BY-NC-ND 4.0) License. 